\title{
Route, Modulation Format, MIMO and Spectrum Assignment in Flex-Grid/MCF Transparent Optical Core Networks
}

\author{
Rubén Rumipamba-Zambrano, Jordi Perelló and Salvatore Spadaro
}

\begin{abstract}
In this paper, we target an optimal Multiple-Input Multiple-Output Digital Signal Processing (MIMO-DSP) assignment to super-channels affected by inter-core crosstalk (ICXT) in Multi-Core Fiber (MCF)-enabled transparent optical core networks. MIMO-DSP undoes ICXT effects, but can be costly with high core density MCFs. Hence, its implementation in the network must be carefully decided. We address our objective as a joint Route, Modulation format, MIMO and Spectrum Assignment (RMMSA) problem, for which Integer Linear Programming (ILP) formulations are provided to optimally solve it in small network scenarios. Moreover, several heuristic approaches are also proposed to solve large-scale problem instances with good accuracy. Their goal is to minimize both network spectral requirements and the amount of MIMO equalized super-channels, taking a crosstalk-free Space Division Multiplexing (SDM) solution as a reference, for example, based on parallel single mode fibers (i.e., a Multi-Fiber-MF-scenario). For our evaluation, we consider several state-of-the-art MCF prototypes and different network topologies. The obtained results, with the considered MCFs, disclose that in national backbone networks, the desirable percentage of super-channels with MIMO equalization to match the performance of an equivalent crosstalk-free SDM solution ranges from $0-36 \%$, while in continental-wide networks this range raises up to $0-56 \%$. In addition, in the case of a non-ideal MIMO (with a $3 \mathrm{~dB} / \mathrm{km}$ of crosstalk compensation), such percentages range from $0-28 \%$ and $0-45 \%$ in national and continental-wide backbone networks, respectively, experimenting a performance gap up to $12 \%$ with respect to the MF reference scenario.
\end{abstract}

Index Terms-Optical networks, Flex-Grid, Space Division Multiplexing, Multi-Core Fibers, MIMO.

\section{INTRODUCTION AND RELATED WORK}

$\mathrm{M}$ ULTI Core Fibers (MCFs) have arisen as promising and economically attractive candidates to realize Space Division Multiplexing (SDM) in ultra-high bit-rate optical core networks [1], [2], thanks to the very low inter-core crosstalk (ICXT) that they introduce during the optical signal propagation, even with high core density [3], [4]. Additionally, Elastic Optical Networks (EONs) [5], making use of a flexible spectrum grid (Flex-Grid) allow taking full

Manuscript received

R. Rumipamba-Zambrano, J. Perelló and S. Spadaro are with the Advanced Broadband Communications Center (CCABA), Universitat Politècnica de Catalunya (UPC) - Barcelona Tech, 08034 Barcelona, Spain (e-mail: rrumipam@ac.upc.edu). advantage of the vast amount of spectral resources provided by future SDM-EON networks. In particular, Flex-Grid/MCF networks have been advocated by many works in the literature as a promising implementation of SDM-EONs [6]-[9].

The new space dimension enables different super-channel (SCh) allocation options [10]. Among them, spatial SChs stand out, as they foster cost reductions at the transceivers by enabling joint digital signal processing [11] and integration [12]. Moreover, spatial SChs allow simplifying the design of SDM-Reconfigurable Optical Add/Drop Multiplexers (ROADMs), opening the possibility to jointly switch their allocated spectrum portion across the entire spatial dimension at once, namely, applying joint-switching (JoS) [13]. That is, all cores can be seen as a single entity.

In MCF-enabled networks, the coupling between cores, i.e., ICXT originating by the continuity of the electromagnetic field in adjacent dielectric regions [14], is an important aspect to deal with. To overcome its negative effects, several strategies focused on ICXT management [6], [8], [15] and suppression by using Multiple-Input Multiple-Output (MIMO) equalization [16], [17] have been proposed in the literature. MIMO-based ICXT suppression through Digital Signal Processing (DSP) can be implemented at the receiver end, as an extension of the $2 \times 2$ MIMO for Polarization Multiplexing (PM) [18]. MIMO-DSP mitigates the negative effects of ICXT; however, its complexity [18], [19] and power consumption [20] are important aspects to be considered. Throughout this paper, we will refer to MIMO for ICXT suppression solely. Note that the overall complexity order including the decoupling of both ICXT and polarization would be $2 \cdot N \times 2 \cdot N$, being $N$ the number of coupled MCF cores.

MIMO-DSP helps recovering several signals coupled together, if all of them are jointly processed at a common receiver. So, in case of MCFs, if two or more cores are adjacent and they transmit optical signals on the same wavelength, ICXT between them can be suppressed if they are co-destined and MIMO is applied. Otherwise, the accumulated ICXT along the routing path has to be kept below a given threshold to properly recover them at the receiver [21].

Saitoh et al. [22] classify MCFs in two categories, namely, weakly- and strongly-coupled MCFs. Both categories are basically defined by the core pitch $(\Lambda)$ and coupling coefficient $(\kappa)$. Weakly-coupled ones have a typical $\Lambda$ higher than $30 \mu \mathrm{m}$ and $\kappa$ lower than $10^{-2} \mathrm{~m}^{-1}$, keeping ICXT below - 
$30 \mathrm{~dB}$ per $100 \mathrm{~km}$ (i.e., $-50 \mathrm{~dB} / \mathrm{km}$ ) [23]. In contrast, stronglycoupled MCFs present lower $\Lambda$ and higher $\kappa$ values, mandating MIMO equalization. Consequently, spatial SChs and JoS become mandatory as well. In this paper, we focus on the analysis of weakly-coupled MCFs, where MIMO becomes optional. In fact, when a single robust (e.g., BPSK) modulation format is considered, e.g., as in traditional Wavelength Division Multiplexing (WDM) networks, ICXT likely does not affect the network Grade-Of-Service (GoS) making MIMO unnecessary. However, when high-order or distance-adaptive modulation formats are considered, like in Flex-Grid networks, significant performance gaps between Multi-Fiber (MF) -a crosstalk-free SDM fiber based on parallel single-mode fibers within the same fiber ribbon cableand MCF solutions can appear. The fact is that, although low ICXT is present, it forces to employ more robust but less spectrally efficient modulation formats over long distance paths affecting the network GoS, as demonstrated in [9], [24]. Hence, the feasibility and convenience of MIMO equalization to compensate ICXT is an interesting case study from a network planning perspective.

On the first matter of feasibility, as stated before, MIMO can be applied if the coupled signals are co-destined. For instance, in [17] it is assumed a network with 12-core MCFs, where lightpaths are carried on one routing path, using a single core and wavelength. Those coupled lightpaths directed to a common destination are equalized, while for the rest of them the accumulated ICXT threshold cannot be surpassed. This makes that the overall network performance (in terms of GoS) cannot approach the one of a crosstalk-free SDM fiber system, even though MIMO is applied. Regarding the convenience of using MIMO, as introduced before, JoS allows switching a spectrum portion across all spatial channels at once (i.e., all sub-channels are co-destined), being possible to apply MIMO to all lightpaths (spatial SChs in this work). However, depending on the XT tolerance, applying MIMO to all lightpaths may not be necessary. So, in this scenario, one important question that arises is: Which lightpaths would be convenient to be equalized in order to reduce the performance gap versus a crosstalk-free SDM benchmark solution?

To the best of our knowledge, this work answers for the first time the question posed above for JoS-enabled FlexGrid/MCF networks. We address the MIMO assignment jointly with the route, modulation format and spectrum assignment (RMSA) problem arising in EON networks, thus defining and targeting a new problem called route, modulation format, MIMO and spectrum assignment (RMMSA). To this aim, we propose an optimal solution based on two Integer Linear Programming (ILP) formulations, and several suboptimal solutions by means of heuristics. The rest of the paper is organized as follows. Section II describes the RMMSA problem. ILP formulations for the joint RMMSA optimization are presented in Section III, while section IV describes the proposed heuristics. Section V presents the numerical results, divided in four subsections. Subsection V.A describes the scenario details and assumptions. The performance evaluation of the proposed heuristics is presented in subsection V.B, whereas the relationship between MIMO equalization and spectral requirements is discussed in subsection V.C in largescale Flex-Grid/MCF optical core networks. Subsection V.D discusses MIMO complexity and crosstalk compensation aspects assuming a non-ideal MIMO. Finally, section VI draws up the main conclusions of this work and envisions future research lines.

\section{Problem Statement}

The targeted RMMSA problem in JoS-enabled FlexGrid/MCF optical core networks can be formally stated as:

Given:

1) A Flex-Grid/MCF network represented as a directed graph $\mathcal{G}(\mathcal{N}, \mathcal{E})$, where $\mathcal{N}$ is the set of optical nodes and $\mathcal{E}$ the set of unidirectional MCF links. All MCF links are assumed of $|\mathcal{C}|$ weakly-coupled cores with identical core layout.

2) A spectral grid consisting of an ordered set of Frequency Slots (FSs), denoted as $\mathcal{S}$, available in every MCF core. FSs have a spectral width (in $\mathrm{Hz}$ ) equal to $W$.

3) A set of admissible modulation formats to be employed by node transceivers, denoted as $\mathcal{M}$. For a given $m \in$ $\mathcal{M}, e f_{m}$ denotes its efficiency (in bits $/ \mathrm{s} \cdot \mathrm{Hz}$ ).

4) A set of offered unidirectional demands to the network, denoted as $\mathcal{D}$. Each $d \in \mathcal{D}$ has associated a source $\left(s_{d}\right)$ and a destination $\left(t_{d}\right)$ node in $\mathcal{G}(\mathcal{N}, \mathcal{E})$ and a requested bit-rate (in $\mathrm{b} / \mathrm{s}$ ), denoted as $r_{d}$.

Find: a lightpath with or without MIMO equalization for supporting every offered demand in $\mathcal{D}$, subject to the following constraints:

1) Spectrum contiguity: spatial SChs must be allocated over a contiguous subset of FSs, the same in all cores of the traversed MCFs (i.e., they use the entire spatial dimension, so that JoS can be applied).

2) Spectrum continuity: in absence of spectrum conversion and regeneration (i.e., transparent transmission), FSs supporting a spatial SCh must be the same in all MCFs along the path from $s_{d}$ to $t_{d}$.

3) Spectrum clashing: a given FS in any core of any MCF can only be allocated to one spatial SCh at most.

4) Maximum number of lightpaths with MIMO $(\eta)$ : in order to save DSP complexity and cost, this is the maximum amount of lightpaths to which MIMO-based ICXT suppression can be applied.

Objective: minimize the network spectral requirements by deciding on the most appropriate route, modulation format, MIMO necessity and spectrum assignment for all lightpaths needed to serve all demands.

The objective of the RMMSA problem is to allocate all demands, deciding on which lightpaths should be equalized by using MIMO to approach the performance (in terms of spectral requirements) of an equivalent MF scenario. Without loss of generality, we can assume that a network operator can 
afford a maximum number of lightpaths with MIMO $\eta$ in the network, $0 \leq \eta \leq|\mathcal{D}|$. When $\eta=0$, none of the lightpaths are equalized using MIMO and the maximum reach (in $\mathrm{km}$ ) of the optical signals is always limited by the accumulated ICXT and the OSNR [9], as in a basic MCF optical network without MIMO-based ICXT suppression. Conversely, by applying MIMO at the receiver end to $\eta>0$ lightpaths, ICXT can be compensated. Assuming an ideal MIMO (i.e., full ICXT suppression), the maximum reach restriction due to ICXT does not apply to those lightpaths anymore, being only limited by OSNR, like in the equivalent MF-based solution. Meanwhile, if a non-ideal MIMO suppression is implemented, the remaining ICXT needs to be evaluated if will or will not prevent an appropriate network performance. This raises the issue to investigate the trade-off between spectrum and MIMO requirements, which will be a key objective of this work. Indeed, there can exist an intermediate $\eta$ value, $\eta=x$, $x \leq|\mathcal{D}|$, where identical spectral requirements as in an equivalent MF scenario can be achieved (assuming an ideal MIMO). We call this $\eta$ value as the sweet spot throughout the paper. As a matter of fact, some candidate lightpaths can trivially be discarded as candidates for MIMO from the very beginning, as they eventually require the same spectral resources with or without MIMO-based ICXT suppression. For instance, in Tab. II (presented later on in section V), let us consider a path with a physical length of $800 \mathrm{~km}$ over 22-core MCFs with identical ICXT characteristics as the MCF prototype reported in reference [25]. There, the most efficient modulation format for both MF (where transmission reach is only limited by OSNR) and MCF (considering ICXT as the overall transmission reach limiting factor) scenarios is the same, i.e., 16-QAM. Consequently, one lightpath served over this path, either in that MCF or equivalent MF scenario would require the same spectrum, making MIMO unnecessary.

\section{OPTIMAL PROBLEM SOLUTION}

In this section, we present two ILP formulations to optimally solve the stated RMMSA problem. Using the first formulation (ILP1), the minimum number of FSs to serve all demands in $\mathcal{D}$ permitting at most $\eta$ lightpaths with MIMO is found. The second formulation (ILP2) is used to find the minimum number of $\eta$ lightpaths with MIMO required to achieve the same spectral requirements as in the equivalent MF solution. It is worth mentioning that ILP2 is not mandatory to solve ILP1, but can speed up the search for the sweet spot $(\eta=x)$.

The following sets and input parameters have been defined for both ILP formulations:

- $\mathcal{P}_{d}$ : Set of pre-computed candidate physical paths for demand $d \in \mathcal{D} ; l_{p}$ is the physical length (in $\mathrm{km}$ ) of path $p$ and $h_{p}$ the number of hops that it traverses.

- $\quad \mathcal{P}$ : Set of all pre-computed candidate physical paths in the network $\left(\mathcal{P}=\cup_{d \in \mathcal{D}} \mathcal{P}_{d}\right)$.

- $\mathcal{L}_{d}$ : Set of pre-computed candidate lightpaths for supporting demand $d \in \mathcal{D}$.

- $\mathcal{L}$ : Set of all candidate lightpaths in the network
$\left(\mathcal{L}=\cup_{d \in \mathcal{D}} \mathcal{L}_{d}\right)$. We also denote as $\mathcal{L}_{e}$ those lightpaths traversing MCF link $e$, and $\mathcal{L}_{e}^{S}$ accounts for lightpaths traversing link $e$ and using slot $s \in \mathcal{S}$.

- $\delta_{l}$ : Binary parameter, equal to 1 if MIMO is applied to candidate lightpath $l, 0$ otherwise. $l^{(1)}$ and $l^{(0)}$ account for lightpath $l$ with and without MIMO, respectively.

- $\quad m_{l^{(1)}}, m_{l^{(0)}}$ : Most efficient modulation format assigned to lightpath $l$ with and without MIMO, respectively.

- $\quad n F S_{l^{(1)}}, n F S_{l^{(0)}}$ : Number of FSs assigned to lightpath $l$ with and without MIMO, respectively.

- GB: Guard-band width (in $\mathrm{GHz}$ ) needed between adjacent spatial SChs to facilitate their filtering.

- $\quad W$ : Spectral grid granularity (i.e., $12.5 \mathrm{GHz}$ according to the ITU-T recommendation [26]).

\section{A. Candidate lightpath pre-computation}

The allocation of a lightpath in the network requires deciding on the physical path that it will traverse from source to destination and the spectrum portion used. Moreover, in our scenario, we assign another attribute $\delta_{l}$ to lightpaths, indicating whether MIMO is applied to them or not. To let both ILP formulations decide on the RMMSA for each demand to be allocated in the network, we rely on the precomputation of candidate lightpaths (i.e., candidate physical paths and candidate frequency slots with/without MIMO). Algorithm 1 details the pseudo-code to this end.

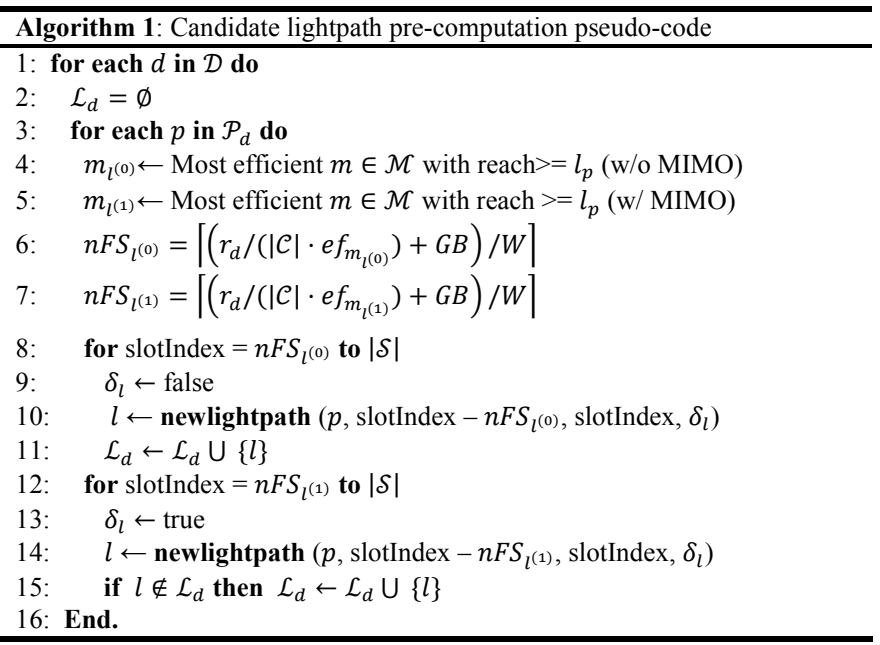

In Algorithm 1, for every demand $d \in \mathcal{D}$ and path $p \in \mathcal{P}_{d}$, the most efficient modulation format whose reach is equal or larger than $l_{p}$, either without $\left(m_{l^{(0)}}\right)$ or with MIMO equalization $\left(m_{l^{(1)}}\right)$ is selected. Then, assuming such modulation formats, the spectral width in FSs of a (candidate) lightpath $l$ over that path $p$, either without $\left(n F S_{l^{(0)}}\right)$ or with MIMO $\left(n F S_{l^{(1)}}\right)$ is obtained (line 4 and 5). Note that, in order to minimize the required spectral width, we assume that the entire spatial dimension of the MCFs is used to configure a spatial SCh, similarly as in the Full Core Assignment (FCA) strategy presented in [9]. Thus, each core supports $r_{d} /|\mathcal{C}| \mathrm{b} / \mathrm{s}$. This latter aspect makes a JoS-enabled Flex-Grid/MCF network equivalent to a Flex-Grid over single spatial channel from the routing point of view. Otherwise, the selected core 
index $i$-th must be also indicated in the lightpath $l$ definition. Next, we find, in a sliding window fashion, all candidate lightpaths of $n F S_{l^{(0)}}$ adjacent FSs over that path, which are added to $\mathcal{L}_{d}$, namely, the set of candidate lightpaths without MIMO to carry demand $d$. The same is done to find all of them with MIMO, that is, of $n F S_{l^{(1)}}$ adjacent FSs. If there exist candidate lightpaths with and without MIMO that require the same number of FSs, we only keep in $\mathcal{L}$ those without MIMO equalization (MIMO is unnecessary).

As a last remark, note that all pre-computed candidate lightpaths already ensure both the spectral contiguity and continuity constraints pointed out in previous section (i.e., they employ the same set of contiguous FSs in all MCF links along the physical path $p$ ).

\section{B. ILP formulations}

For both ILP formulations, the following decision variables are introduced:

- $x_{l}$ : Binary; equal to 1 if candidate lightpath $l \in \mathcal{L}_{d}$ is used to allocate demand $d \in \mathcal{D}$; 0 otherwise.

- $y_{e s}$ : Binary; equal to 1 if FS $s \in \mathcal{S}$ is used in link $e \in \mathcal{E} ; 0$ otherwise.

- $y_{s}$ : Binary; equal to 1 if FS $s \in \mathcal{S}$ is used in any link of the network; 0 otherwise.

The ILP1 formulation to quantify the minimum number of FSs needed to serve all demands in $\mathcal{D}$ reads:

$$
\text { minimize } \Phi=\sum_{s \in \mathcal{S}} y_{s}
$$

subject to:

$$
\begin{gathered}
\sum_{l \in \mathcal{L}_{d}} x_{l}=1, \forall d \in \mathcal{D} \\
\sum_{l \in \mathcal{L}_{e}^{s}} x_{l} \leq y_{e s}, \forall e \in \mathcal{E}, s \in \mathcal{S} \\
\sum_{e \in \mathcal{E}} y_{e s} \leq|\mathcal{E}| y_{s}, \forall s \in \mathcal{S} \\
\sum_{l \in \mathcal{L}} \delta_{l} \cdot x_{l}=\eta
\end{gathered}
$$

Objective function (1) aims to minimize the total number of FSs used in any MCF link in the network (i.e., $\Phi$, considered hereafter as the spectrum usage metric). Constraint (2) enforces that every offered demand is supported over a single lightpath. Constraint (3) is the spectrum clashing constraint, which ensures that any FS in any MCF link supports one lightpath at most. Constraint (4) assigns the value of variables $y_{s}$. Finally, constraint (5) limits the number lightpaths with MIMO to $\eta$.

Thus, by sweeping $\eta \in[0,|\mathcal{D}|]$ we can find the relationship between MIMO equalization and spectrum usage. One should expect that the higher the number of lightpaths with MIMO, the lower the number of required spectral resources. The upper $\left(\Phi_{M C F}\right)$ and lower $\left(\Phi_{M F}\right)$ bounds of the objective function $(\Phi)$ can be obtained if constraint (5) is not considered and candidate lightpath computation in algorithm 1 only includes the ones employing $m_{l^{(0)}}$ (i.e., without MIMO) or $m_{l^{(1)}}$ (i.e., assuming an ideal MIMO) modulation formats, respectively. Conversely, in order to compute the spectral requirements for $0<\eta<|\mathcal{D}|$ values, it is necessary that set $\mathcal{L}$ includes all candidate lightpaths with and without MIMO equalization.
Moreover, in order to find out the sweet spot $(\eta=x)$, where the spectrum utilization is the same as in the MF equivalent solution, ILP1 can be adapted as follows (ILP2):

$$
\text { minimize } \eta=\sum_{l \in \mathcal{L}} \delta_{l} \cdot x_{l}
$$

subject to constraints: (2), (3), (4), and

$$
\sum_{s \in \mathcal{S}} y_{s} \leq \Phi_{M F}
$$

In ILP2, objective function (6) aims to minimize the number of lightpaths with MIMO. Moreover, note that the value of ILP1 objective function for the MF solution $\left(\Phi_{M F}\right)$ feeds as an input parameter in constraint (7), in order to enforce that the total number of FSs used in any link does not exceed the value found for the MF-based solution. Therefore, ILP2 targets the opposite objective of ILP1.

\section{HEURISTIC APPROACHES}

In this section, we introduce several heuristics in order to solve the RMMSA problem. It has been widely demonstrated in the literature that the RSA problem is Non-deterministic Polynomial (NP)-hard [27][28], meaning, in few words and very informally, that the problem cannot be optimally solved in polynomial (deterministic) time by means of an algorithm. Furthermore, the additional modulation format and MIMO assignment sub-problems present in RMMSA increase its computational complexity of RSA even more. For this reason, for large-scale problem instances, it is necessary to provide sub-optimal approaches employing heuristics in order to solve the RMMSA problem in polynomial times. In this work, we propose two greedy heuristics (i.e., algorithms choosing the partial solution that looks the best one at each stage, thus providing myopic or locally optimum solutions, not necessarily globally optimum) inspired in the Balanced Load Spectrum Allocation (BLSA) and Maximum Reuse Spectrum Allocation (MRSA) heuristics presented in [29]. Moreover, a third greedy heuristic is proposed, which selects from the set of candidate lightpaths the one with the lowest indexed starting FS, similarly to the RMSA heuristic presented in [27]. We call this latter heuristic as Lowest Indexed Spectrum Allocation (LISA). Our contribution is the introduction of the MIMO assignment sub-problem into these three greedy heuristics. These heuristics serve to obtain an initial solution of the addressed RMMSA problem instance, which is later on improved by means of a simulated annealing (SA)-based meta-heuristic (SA-RMMSA), thus further approaching to the global optimum solution. Algorithms 2 and 3 show the pseudo-code of these greedy heuristics. As BLSA and LISA only differ in the routing path selection, we present both heuristics in algorithm 2. Meanwhile, the MRSA heuristic is presented in algorithm 3.

According to algorithm 2, for each demand $d \in \mathcal{D}$ we select from $\mathcal{P}_{d}$ (e.g., $K=3$ shortest paths (SPs)) the least congested (LC) or lowest indexed starting FS (LISFS) path $p_{j}$ for BLSA and LISA, respectively. The rest of the code performs most spectrally efficient (MSE) modulation format assignment and First-Fit (FF) MIMO and spectrum assignment. For the MIMO assignment, we pre-compute the candidate demands to be served with MIMO $\left(\mathcal{D}_{\text {MiMo_Candidates }} \subseteq \mathcal{D}\right)$ by discarding MIMO 
equalization for demands whose candidate paths report the same spectral requirements with or without it.

Regarding the MIMO assignment, we define a fitness function $(f)$ as the savings (in terms of FSs) achievable by allocating a lightpath over the selected path $p_{j}$ with MIMO $\left(n F S_{1}\right)$ versus without MIMO equalization $\left(n F S_{2}\right)$, multiplied by the number of hops of $p_{j}$, namely, $f=\left(n F S_{2}-n F S_{1}\right) \cdot h_{p_{j}}$. Thus, the minimum $f$ value is 0 (i.e., being the required FSs for a lightpath identical with or without MIMO), while its maximum value ( $\max$ ) can be computed for the longest shortest path (i.e., for the network diameter). In order to determine the goodness of assigning MIMO equalization to a lightpath, each computed $f$ value has to be compared with a reference value $(V) . V$ is defined as the highest fitness value between 0 and $\max$, namely, $V \in\left\{\mathbb{Z}^{+} \mid 0 \leq V \leq \max \right\}$ that allows getting at least $\eta$ requested lightpaths with MIMO. $V$ is pre-computed at the beginning of each simulation by decrementing it from $\max$ to 0 . We run the heuristic for each $V$ value and find the number of potential lightpaths to be served with MIMO. If this number is higher than $\eta$ then we stop and set $V$. If $V$ is very low, the goodness of the MIMO equalization could be poor, while if it is very high, it could occur that MIMO assignment is extremely restrictive. Before deciding on the MIMO assignment, each demand $d$ is firstly checked if it belongs to $\mathcal{D}_{\text {Mimo Candidates }}$ and if the number of allocated lightpaths with MIMO $\left(l_{\text {mimo }}\right)$ is lower than $\eta$ (line 9 ). If so, the fitness function is analyzed. Otherwise, the number of FSs in the MCF scenario without MIMO is considered for lightpath allocation. Lightpaths whose $f$ value is higher than $V$ (line 10) are equalized at the receiver. Finally, demand $d$ is accommodated (line 21) in the network using path $p_{j}$, over $n F S$ contiguous FSs, with/without MIMO (1/0).

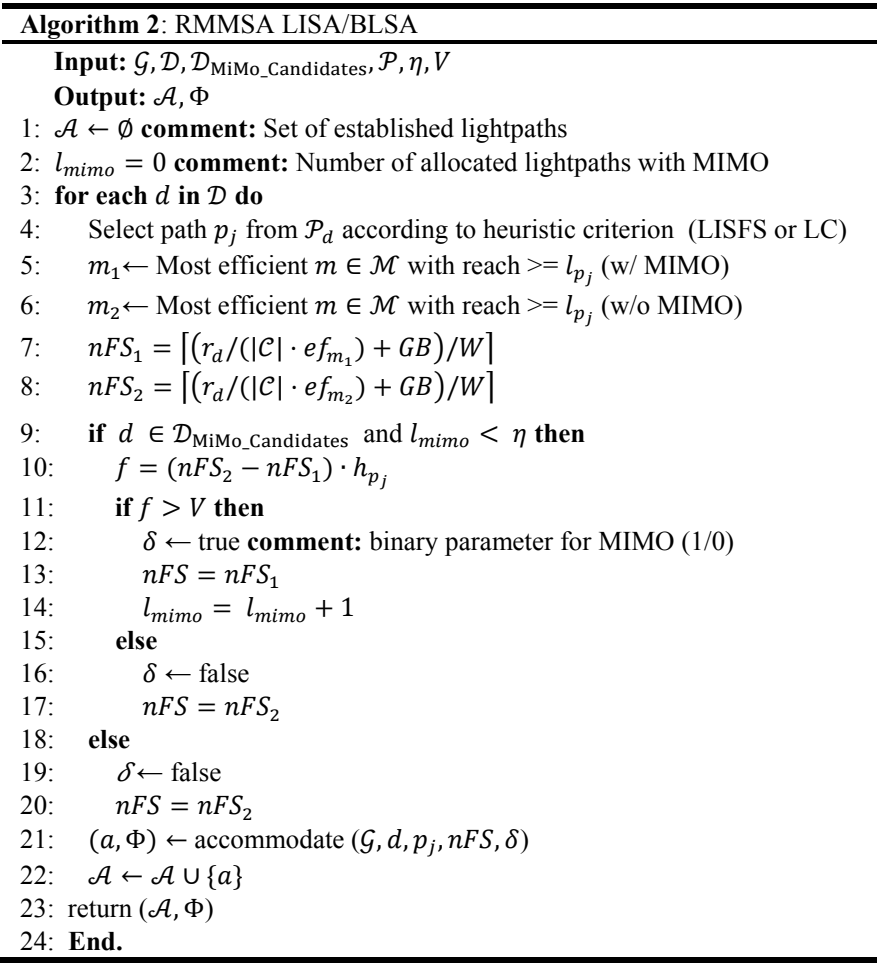

Regarding algorithm 3, two iterative processes are followed to allocate lightpaths over the shortest disjoint path (SDP) in the first available and consecutive FSs. In each iteration $j$ of the outer loop (line 2), the first pending demand in $\mathcal{D}$ is served over the shortest path $p_{j}$ on the original network graph $\mathcal{G}$, computed, e.g., by means of the Dijkstra algorithm. After that, in the inner loop (line 7) each pending demand $d \in \mathcal{D}$ is served over the shortest path $p_{i}$, which is also disjoint with all already established routing paths $\mathcal{R}$ (line 8) in the current outer loop iteration $j$. The demands, for which finding a SDP or available spectrum portion in the current iteration $j$ is impossible, have the opportunity to be accommodated in subsequent iterations. As in algorithm 2, MSE modulation format, FF MIMO and spectrum assignment are considered. Then, steps from 5 to 22 in algorithm 2 are executed after the routing path selection. Again, MIMO assignment is based on fitness function $f$.

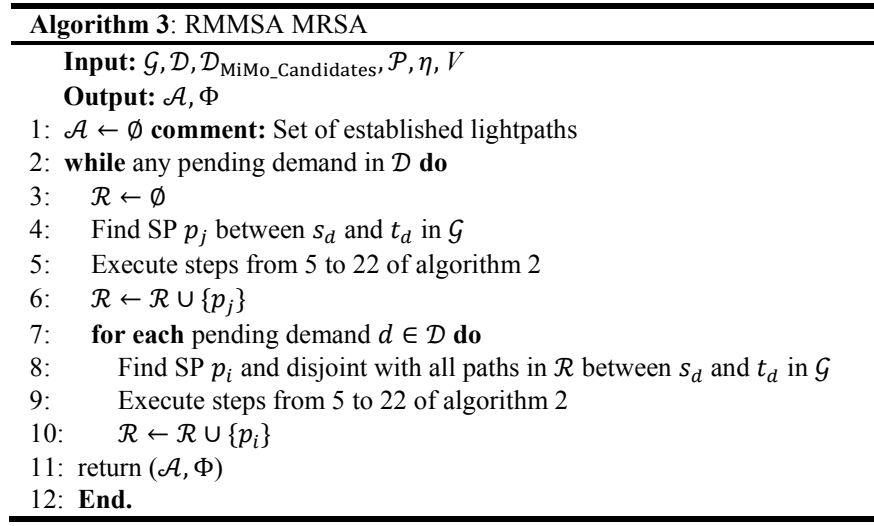

As for SA-RMMSA (algorithm 4), there are three versions of it, employing each one of the presented greedy heuristics. In fact, lines 4, 13 and 23 consist in the evaluation of the objective function by running LISA, BLSA or MRSA heuristics. The SA meta-heuristic is inspired in the annealing processes to produce crystals [30]. We use this meta-heuristic to change the order of elements in $\mathcal{D}$ aimed at improving objective function $\Phi$. The initial solution $\left(\omega_{o}\right)$ is obtained sorting the demands in $\mathcal{D}$ in descending order, according to their required number of FSs over their shortest path. SA is also known as a local search-based meta-heuristic, whose solutions evolve in the neighborhood $\left(\omega^{\prime}\right)$ of the current solution $(\omega)$ not only by accepting improving solutions (like, hill climbing movements), but also worse solutions (uphill movements) to provide diversification within the solution space. A neighboring solution $\left(\omega^{\prime}\right)$, as intensification strategy, is defined as a swap movement (line 11) between two randomly chosen demands of the current order $(\omega)$ in $\mathcal{D}$. Thus, the process starts with an initial temperature $T(0)$ and it continues decreasing it in each iteration (line 20) with a cooling rate $\alpha \in(0,1), \alpha=0.9$ for us. The temperature affects the acceptance probability $\left(P_{a c c}\right)$ of non-improving solutions. In fact, the $P_{a c c}$ depends on the objective function worsening $\left(\Omega=\Phi_{\omega^{\prime}}-\Phi_{\omega}\right)$ and the temperature, namely, $P_{a c c}=e^{-\Omega / T}$. For example, we initially decide to accept with probability 0.3 a solution yielding an objective function worsening in one FS. Then, $e^{-1 / T(0)}=0.3$, and $T(0)=-1 / \ln (0.3)$-line 6-. After the evaluation of each neighboring solution $\left(\omega^{\prime}\right)$, if $\omega^{\prime}$ 
improves the resulting $\Phi$ value of the incumbent solution $\left(\omega^{*}\right)$, then this is updated $\left(\omega^{*}=\omega^{\prime}\right.$, line 17$)$. This process ends after 20000 iterations (maxIter) or when the temperature reaches freezing state (e.g., 0). Finally, note that by changing the order of demands, different solutions for MIMO assignment can also be explored, as this resource is assigned on a FF basis.

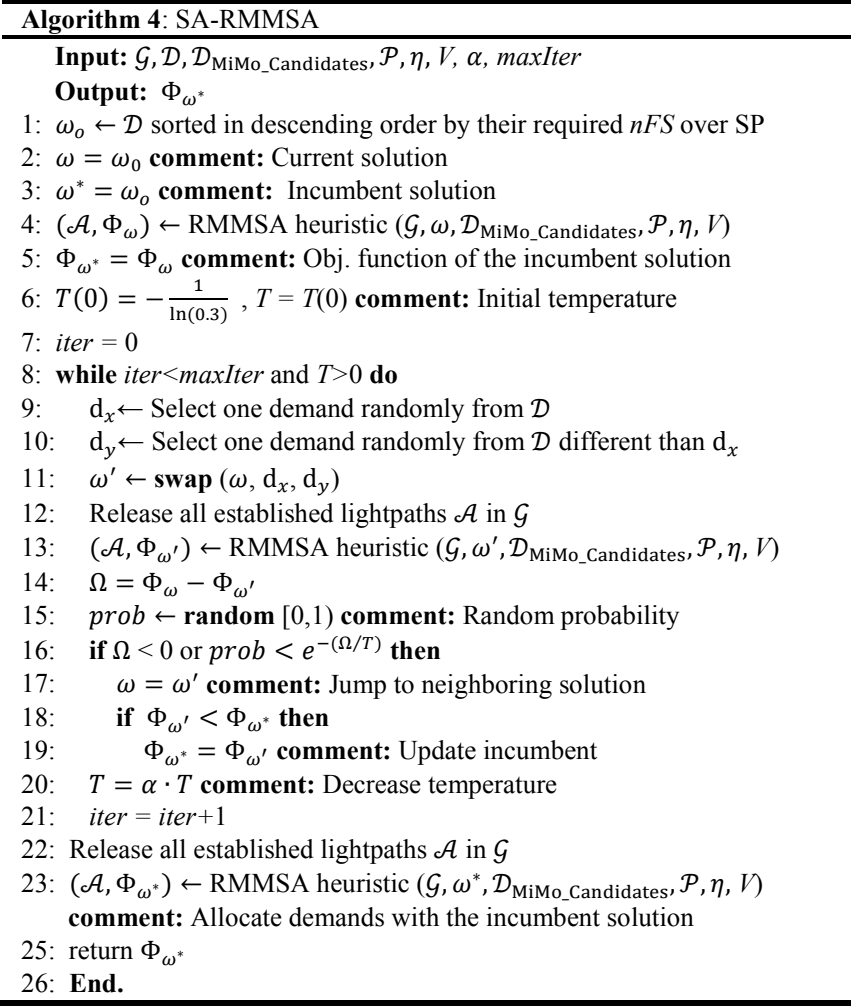

\section{NUMERICAL RESULTS}

This section presents the performance evaluation of the proposed solutions to solve the RMMSA problem. To this end, we firstly detail the evaluation scenarios and assumptions. Next, we validate the performance of our proposed SA-based meta-heuristics against the optimal solution of ILP1. Finally, we evaluate the MIMO equalization and its spectral usage benefits in two large-scale optical core network scenarios using the proposed SA-based meta-heuristics.

\section{A. Scenario Details and Assumptions}

We consider the different topologies shown in Fig. 1, whose main characteristics are depicted in Tab. I. In order to evaluate the performance of SA-based heuristics against ILP1, we use two test topologies (Fig. $1(\mathrm{a}, \mathrm{b})$ ) of 6 and 9 nodes. Meanwhile, for larger problem instances, we consider the National 12node Deutsche Telekom optical network (DT12, Fig. 1(c)) and the 15-node National Science Foundation Network (NSF15, Fig. 1(d)). For the experiments carried out in next subsections, we consider that each network link is equipped with one weakly-coupled MCF of $|\mathcal{C}| \in\{7,12,19,22,30\}$, whose characteristics match those of real MCF prototypes found in the literature [25], [31]-[34]. These reported prototypes are the best single-mode MCFs (in terms of ICXT) and they have been selected in line with our previous work [24], [35]. The

measured worst aggregate ICXT of these MCF prototypes is shown in Tab. II.

As for the transmission reach (TR) of the optical signals, we adopt the method for MCF-enabled networks presented in [9], which we summarize here to help the reader better understanding the outcome of this work. Specifically, the overall TR is considered to be the minimum between the one imposed either by intra- or inter-core impairments (which is also assumed in [36]). As for the TR limitation imposed by intra-core impairments, the values provided by the GN-model under "Link 1" [37] are employed, which considers ASE noise and nonlinear interference (NLI) as independent and additive Gaussian-like noises at least for low-to-moderate nonlinearity. The GN-model determines the optimum launch power per channel maximizing the OSNR (therefore, the TR) for several modulations formats. Note that low optical power levels in transport networks decrease nonlinear effects. The linear impairments, such as chromatic and polarization dispersion, can be electronically compensated by means of DSP capabilities of coherent detectors, so that they are not considered as TR limiting factors. Meanwhile, regarding the TR limitation imposed by inter-core impairments (i.e., ICXT), the maximum in-band crosstalk tolerated by each modulation format for a $1 \mathrm{~dB}$ Optical Signal-To-Noise Ratio (OSNR) penalty is considered [38]. One important aspect to be emphasized, in the case of weakly-coupled MCFs, is that ICXT can be treated as independent of NLI. In fact, some models (e.g., see [39], [40]) consider ICXT effect as an additional and independent Gaussian-like noise source. Therefore, they include ICXT as an additive Gaussian noise in the calculations of OSNR. For more details about the TR estimations, please see reference [9]. Tab. III shows the TR limitation imposed by OSNR (using the GN-model), ICXT, as well as the overall TR for different MCFs and modulation formats. Looking at the overall TR values, we can appreciate that ICXT is not the limiting factor in 7- and 12-core MCFs, but it is in 19-, 22- and 30-core MCFs. Therefore, we do not consider in next subsections the 7- and 12-core MCFs since they do not require MIMO crosstalk compensation (i.e., $0 \%$ of MIMO requirements).

(a)

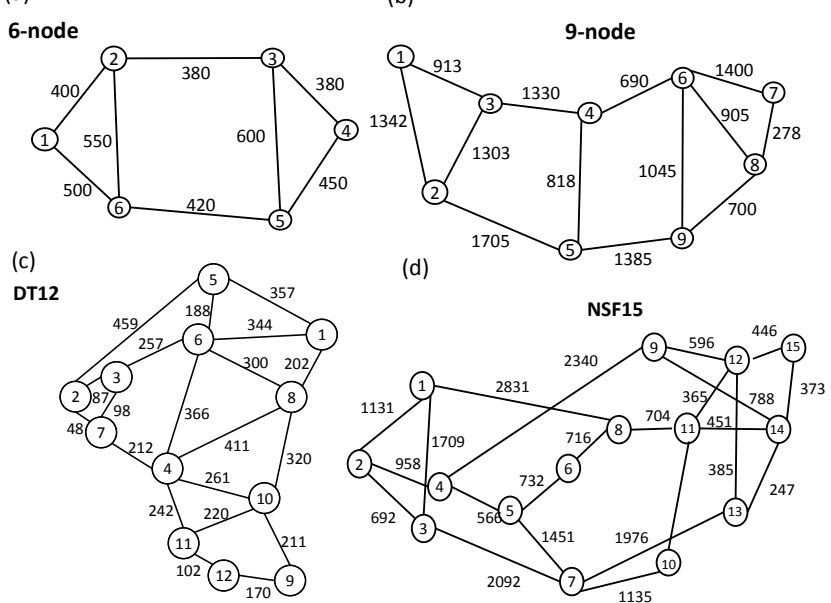

Fig. 1. Reference networks (a) 6-node topology, (b) 9-node topology, (c) DT12 and (d) NSF15. 
TABLE I

MAIN CHARACTERISTICS OF REFERENCE NETWORKS

\begin{tabular}{ccccc}
\hline \hline $\begin{array}{c}\text { Network } \\
(|\mathcal{N}|,|\mathcal{E}|)\end{array}$ & $\begin{array}{c}\text { Avg. Link } \\
\text { Length } \\
{[\mathbf{k m}]}\end{array}$ & $\begin{array}{c}\text { Network } \\
\text { Diameter } \\
{[\mathbf{k m}]}\end{array}$ & $\begin{array}{c}\text { Nodal } \\
\text { Degree } \\
\text { Min/Avg/Max }\end{array}$ & $\begin{array}{c}\text { Network } \\
\text { connectivity }\end{array}$ \\
\hline \hline TEST1 (6, 8) & 460 & 1,160 & $2 / 2.67 / 3$ & 7.88 \\
TEST2 $(9,13)$ & 1,063 & 4,116 & $2 / 2.88 / 4$ & 8.79 \\
DT $(12,20)$ & 243 & 1,019 & $2 / 3.33 / 5$ & 9.96 \\
NSF $(15,23)$ & 1,022 & 4,688 & $2 / 2.88 / 4$ & 9.79 \\
\hline
\end{tabular}

We also consider that each fiber/core has $|\mathcal{S}|=320 \mathrm{FSs}$ (corresponding to $4 \mathrm{THz} \mathrm{C}$-Band discretized by $W=12.5 \mathrm{GHz}$ ). Demands are allocated using spatial SChs with $G B=7.5 \mathrm{GHz}$ [9] and they are switched at ROADMs using JoS. To form the spatial SChs we employ the entire spatial domain. Demands consist of a requested bit-rate $\left(r_{d}\right)$ between source $\left(s_{d}\right)$ and destination $\left(t_{d}\right)$ nodes. Traffic is distributed uniformly between all source-destination node pairs. Moreover, demands request unidirectional connections of bit-rate $r_{d} \in\{400,800$, $1200,1600,2000\} \mathrm{Gb} / \mathrm{s}$ with the same probability of $1 / 5$. The average requested bit-rate per demand is $1.2 \mathrm{~Tb} / \mathrm{s}$. Simulations were run on a 4-core Intel i7 $3.4 \mathrm{GHz}$ PC with $16 \mathrm{~GB}$ RAM, where the IBM ILOG CPLEX v. 12.6 solver [41] was installed and used for solving the ILP formulations.

TABLE II

MEASURED WORST AGGREGATED ICXT IN DB/KM

\begin{tabular}{c|c|c|c|c}
\hline \hline $\mathbf{7}$ cores [29] & 12 cores [30] & 19 cores [31] & 22 cores [23] & $\mathbf{3 0}$ cores [32] \\
\hline-84.7 & -61.9 & -54.8 & -56.2 & -60 \\
\hline
\end{tabular}

TABLE III

TRANSMISSION REACH IN KM

\begin{tabular}{cccccc}
\hline \hline MCF & Limited by & 64-QAM & 16-QAM & QPSK & BPSK \\
\hline \multirow{4}{*}{7 -core } & OSNR & 600 & 2,000 & 9,000 & $>20,000$ \\
& ICXT & 148,098 & 589,589 & $2,347,195$ & $4,683,271$ \\
& Overall & $\mathbf{6 0 0}$ & $\mathbf{2 , 0 0 0}$ & $\mathbf{9 , 0 0 0}$ & $>\mathbf{2 0 , 0 0 0}$ \\
\hline \multirow{3}{*}{ 12-core } & OSNR & 600 & 2,000 & 9,000 & $>20,000$ \\
& ICXT & 769 & 3,062 & 12,190 & 24,322 \\
& Overall & $\mathbf{6 0 0}$ & $\mathbf{2 , 0 0 0}$ & $\mathbf{9 , 0 0 0}$ & $>\mathbf{2 0 , 0 0 0}$ \\
\hline \multirow{3}{*}{ 19-core } & OSNR & 600 & 2,000 & 9,000 & $>20,000$ \\
& ICXT & 150 & 599 & 2,383 & 4,755 \\
& Overall & $\mathbf{1 5 0}$ & $\mathbf{5 9 9}$ & $\mathbf{2 , 3 8 3}$ & $\mathbf{4 , 7 5 5}$ \\
\hline \multirow{3}{*}{22 -core } & OSNR & 600 & 2,000 & 9,000 & $>20,000$ \\
& ICXT & 209 & 832 & 3,311 & 6,607 \\
& Overall & $\mathbf{2 0 9}$ & $\mathbf{8 3 2}$ & $\mathbf{3 , 3 1 1}$ & $\mathbf{6 , 6 0 7}$ \\
\hline \multirow{3}{*}{30 -core } & OSNR & 600 & 2,000 & 9,000 & $>20,000$ \\
& ICXT & 501 & 1,995 & 7,943 & 15,849 \\
& Overall & $\mathbf{5 0 1}$ & $\mathbf{1 , 9 9 5}$ & $\mathbf{7 , 9 4 3}$ & $\mathbf{1 5 , 8 4 9}$ \\
\hline \multirow{2}{*}{. } & & & & &
\end{tabular}

\section{B. Meta-heuristic Performance Validation}

In this subsection, we compare the performance of ILP1 versus the SA-based RMMSA meta-heuristics. To this end, we consider the two MCF prototypes with the highest ICXT (19core and 22-core MCFs) in order to assess the efficiency of meta-heuristics under relevant ICXT levels. Tab. IV to VII show the different values of the objective function $(\Phi)$ for ILP1, as well as for the SA-based metaheuristics (SA-LISA, SA-BLSA, SA-MRSA). The CPLEX solver has been configured to finish the execution and return the result upon reaching an optimality gap lower or equal than $2 \%$ or a maximum execution time of 12 hours. Tab. IV and V show the results in the 6-node topology, while Tab. VI and VII show the results in the 9-node topology. We obtain $\Phi$ for different $\eta$ values in order to observe the influence of (ideal) MIMO equalization in the spectrum usage. $\eta=0$ value represents, as stated in Section II, the results for a MCF-enabled network, where none of the lightpath are allocated with MIMO, while the last $\eta$ value of each table corresponds to sweet spot $x$. This latter value, which yields the minimum spectrum usage, has been obtained using ILP2. Additionally, all tables show the execution times (in min.) for ILP1 and meta-heuristics.

Specifically, for the 6-node topology (results of Tab. IV and $\mathrm{V}$ ), we use a set of 600 and 800 demands $|\mathcal{C}|=19$ and $|\mathcal{C}|=22$, respectively, which represent a medium offered load for different $\eta$ values (lower than $\sim 0.7$ of spectrum occupation). The relative error between the results of the meta-heuristics and those of ILP1 is depicted under the $\Phi$ value. As observed, this error is below $8.3 \%$ (maximum observed one with the SABLSA meta-heuristic) and even $0 \%$ in some cases. Regarding the execution times, ILP1 requires $38 \mathrm{~min}$ in average to find the optimal solution. The SA-based meta-heuristics reduces significantly the execution times down to $1.2 \mathrm{~min}$ in average for SA-LISA. Indeed, among the three meta-heuristics, SALISA presents the lowest execution time, while SA-MRSA the highest one, $4.8 \mathrm{~min}$ in average. SA-BLSA requires intermediate execution times between SA-LISA and SAMRSA. Additionally, for $|\mathcal{C}|=19$ the sweet spot $x$ is obtained with 167 lightpaths allocated with MIMO. The remaining 433 lightpaths do not need MIMO-based ICXT suppression. This means that, MIMO has to be applied to $\sim 28 \%$ of lightpaths in order to meet the performance of the MF-based benchmark solution. As for $|\mathcal{C}|=22$, the percentage of lightpaths with MIMO required to meet the performance of equivalent MF solution is reduced to $\sim 15 \%(121 / 800)$ due to its lower worst aggregate ICXT.

For the 9-node topology (results of Tab. VI and VII), we offer 800 demands for both $|\mathcal{C}|=19$ and $|\mathcal{C}|=22$ MCFs. This topology, with more nodes and links, increases the computational complexity of the ILP formulation. This is reflected in the results of ILP1, where for several $\eta$ values ILP1 is not able to find any solution (cells with dotted lines) or the returned value has optimality gap higher than $2 \%$ (i.e., the ILP1 required more than 12 hours to be solved), which is also shown under the $\Phi$ value in the corresponding column of ILP1. For this reason, the relative error between ILP1 and SAbased meta-heuristics only can be computed for a few cases, where errors lower than $6.6 \%$ are reported. Regarding the execution times, in those cases where optimal solutions were obtained, ILP1 requires up to 9.4 hours to find them. However, in most of the cases, after running for 12 hours, ILP1 does not return any solution. This situation occurs mostly for $|\mathcal{C}|=19$. The lower computational complexity with 22-core MCF can be explained not only by the fact that demands may require lower spectral resources facilitating the spectrum assignment sub-problem, but also because the ICXT introduced by 19 -core MCFs is higher. Hence, as candidate lightpaths with and without MIMO require different number of FSs, the former ones cannot be removed from $\mathcal{L}_{d}$ (i.e., MIMO equalization can yield benefits in terms of spectral requirements). This latter aspect is more evident in long-haul networks, increasing the cardinality of the set of candidate lightpaths $\mathcal{L}$ (computed by algorithm 1) and, therefore, the number of decision variables. For instance, in the case of 
TABLE IV

PERFORMANCE VALIDATION IN 6-NODE TOPOLOGY WITH 19-CORE MCF

\begin{tabular}{|c|c|c|c|c|c|c|c|c|}
\hline \multicolumn{9}{|c|}{ 6-node Topology, $|\mathcal{C}|=19,|\mathcal{D}|=600$} \\
\hline \multirow[b]{2}{*}{$\boldsymbol{\eta}$} & \multicolumn{4}{|c|}{$\Phi$} & \multicolumn{4}{|c|}{ Exec. Time (min) } \\
\hline & ILP1 & $\begin{array}{l}\text { SA- } \\
\text { LISA }\end{array}$ & $\begin{array}{l}\text { SA- } \\
\text { BLSA }\end{array}$ & $\begin{array}{l}\text { SA- } \\
\text { MRSA }\end{array}$ & ILP1 & $\begin{array}{l}\text { SA- } \\
\text { LISA }\end{array}$ & $\begin{array}{l}\text { SA- } \\
\text { BLSA }\end{array}$ & $\begin{array}{l}\text { SA- } \\
\text { MRSA }\end{array}$ \\
\hline \multirow{2}{*}{0} & 210 & 210 & 210 & 210 & \multirow{2}{*}{48.7} & \multirow{2}{*}{1.3} & \multirow{2}{*}{2.6} & \multirow{2}{*}{3.3} \\
\hline & & $0 \%$ & $0 \%$ & $0 \%$ & & & & \\
\hline \multirow{2}{*}{33} & 195 & 198 & 196 & 197 & \multirow{2}{*}{65.1} & \multirow{2}{*}{1.3} & \multirow{2}{*}{2.8} & \multirow{2}{*}{3.2} \\
\hline & & $1.5 \%$ & $0.5 \%$ & $1 \%$ & & & & \\
\hline \multirow{2}{*}{66} & 187 & 188 & 187 & 188 & \multirow{2}{*}{43.3} & \multirow{2}{*}{1.3} & \multirow{2}{*}{2.6} & \multirow{2}{*}{3.1} \\
\hline & & $0.5 \%$ & $0 \%$ & $0.5 \%$ & & & & \\
\hline \multirow{2}{*}{99} & 178 & 180 & 180 & 181 & \multirow{2}{*}{19.3} & \multirow{2}{*}{1.2} & \multirow{2}{*}{1.9} & \multirow{2}{*}{3.4} \\
\hline & & $1.1 \%$ & $1.1 \%$ & $1.7 \%$ & & & & \\
\hline \multirow{2}{*}{132} & 170 & 172 & 173 & 173 & \multirow{2}{*}{23.2} & \multirow{2}{*}{1.2} & \multirow{2}{*}{1.7} & \multirow{2}{*}{3.4} \\
\hline & & $1.2 \%$ & $1.8 \%$ & $1.8 \%$ & & & & \\
\hline \multirow{2}{*}{167} & 161 & 166 & 167 & 167 & \multirow{2}{*}{36.7} & \multirow{2}{*}{1.1} & \multirow{2}{*}{1.6} & \multirow{2}{*}{2.9} \\
\hline & & $3.1 \%$ & $3.7 \%$ & $3.7 \%$ & & & & \\
\hline
\end{tabular}

TABLE VI

PERFORMANCE VALIDATION IN 9-NODE TOPOLOGY WITH 19-CORE MCF 9-node Topology, $|\mathcal{C}|=\mathbf{1 9},|\mathcal{D}|=800$

\begin{tabular}{|c|c|c|c|c|c|c|c|c|}
\hline \multirow[b]{2}{*}{$\boldsymbol{\eta}$} & \multicolumn{4}{|c|}{$\Phi$} & \multicolumn{4}{|c|}{ Exec. Time (min) } \\
\hline & ILP1 & $\begin{array}{l}\text { SA- } \\
\text { LISA }\end{array}$ & $\begin{array}{l}\text { SA- } \\
\text { BLSA }\end{array}$ & $\begin{array}{l}\text { SA- } \\
\text { MRSA }\end{array}$ & ILP1 & $\begin{array}{l}\text { SA- } \\
\text { LISA }\end{array}$ & $\begin{array}{l}\text { SA- } \\
\text { BLSA }\end{array}$ & $\begin{array}{l}\text { SA- } \\
\text { MRSA }\end{array}$ \\
\hline 0 & -- & 260 & 259 & 261 & -- & 2 & 2.4 & 11.8 \\
\hline 48 & -- & 231 & 232 & 228 & -- & 2.1 & 2.4 & 3.9 \\
\hline 96 & $\begin{array}{l}230 \\
14 \%\end{array}$ & 214 & 216 & 210 & $>12 \mathrm{~h}$ & 1.8 & 2.2 & 3.9 \\
\hline 144 & -- & 204 & 209 & 203 & -- & 1.2 & 2.3 & 5.7 \\
\hline 192 & $\begin{array}{l}200 \\
10 \% \\
\end{array}$ & 193 & 194 & 189 & $>12 \mathrm{~h}$ & 1.2 & 2.3 & 4.3 \\
\hline 244 & -- & 186 & 189 & 183 & -- & 1.2 & 2.3 & 4.4 \\
\hline
\end{tabular}

$|\mathcal{C}|=22$ and $\eta=48$ the number of candidate lightpaths $(|\mathcal{L}|)$ is equal to 519267 , while for $|\mathcal{C}|=19$ this number increases to 708841. SA-based meta-heuristics reduce significantly the execution times, down to $1.7 \mathrm{~min}$ in average with SA-LISA. Like in the 6-node topology, among the three meta-heuristics, SA-LISA presents the lowest execution times, while SAMRSA reports the highest one, $5 \mathrm{~min}$ in average. SA-BLSA requires intermediate execution times between SA-LISA and SA-MRSA. Additionally, for $|\mathcal{C}|=19$, the sweet spot $x$ is obtained with 244 lightpaths served with MIMO. The remaining 556 lightpaths do not need MIMO-based ICXT suppression. This means that, a $\sim 31 \%$ of lightpaths with MIMO are required to meet the performance of the MF-based benchmark solution. When $|\mathcal{C}|=22$, this percentage falls down to $\sim 12 \%(96 / 800)$ due to its lower ICXT. It is worth mentioning that, for $|\mathcal{C}|=19$ the sweet spot $x$ was returned by ILP2 formulation after $12 \mathrm{~h}$ of execution (optimality gap 22\%).

Furthermore, it is interesting to observe the relationship between $\Phi$ and $\eta$. For instance, taking into account optimalsolutions in the 6-node topology, where it has been possible to obtain results for all $\eta$ values, we found that penalties in terms of spectral requirements increases slowly as the number of lightpaths with MIMO reduces. Thus, for $|\mathcal{C}|=19$, a $60 \%$ MIMO reduction (66 vs. 167 ) is translated only into a $16 \%$ increment in spectrum usage (187 vs. $161 \mathrm{FSs})$, and for $|\mathcal{C}|=22$ a $49 \%$ MIMO reduction (121 vs. 62) only entails an $8 \%$ increment in terms of spectrum usage (212 vs. $197 \mathrm{FSs}$ ). This aspect suggests us that the trade-off between MIMO equalization versus spectral requirements (therefore, network GoS) is an important aspect to be analyzed in detail. If the goal is to save computational complexity and power
TABLE V

PERFormance VALIDATION IN 6-NODE TOPOLOGY WITH 22-CORE MCF 6-node Topology, $|\mathcal{C}|=\mathbf{2 2},|\mathcal{D}|=800$

\begin{tabular}{|c|c|c|c|c|c|c|c|c|}
\hline \multicolumn{9}{|c|}{ 6-node Topology, $|\mathcal{C}|=22,|\mathcal{D}|=800$} \\
\hline \multirow[b]{2}{*}{$\boldsymbol{\eta}$} & \multicolumn{4}{|c|}{$\Phi$} & \multicolumn{4}{|c|}{ Exec. Time (min) } \\
\hline & ILP1 & $\begin{array}{l}\text { SA- } \\
\text { LISA }\end{array}$ & $\begin{array}{l}\text { SA- } \\
\text { BLSA }\end{array}$ & $\begin{array}{l}\text { SA- } \\
\text { MRSA }\end{array}$ & ILP1 & $\begin{array}{l}\text { SA- } \\
\text { LISA }\end{array}$ & $\begin{array}{l}\text { SA- } \\
\text { BLSA }\end{array}$ & $\begin{array}{l}\text { SA- } \\
\text { MRSA }\end{array}$ \\
\hline \multirow{2}{*}{0} & 243 & 244 & 243 & 244 & 289 & 12 & 4 & 81 \\
\hline & & $0.4 \%$ & $0 \%$ & $0.4 \%$ & 28.9 & 1.2 & 4 & 8.1 \\
\hline \multirow{2}{*}{31} & 228 & 231 & 229 & 231 & 535 & 12 & 29 & 6 \\
\hline & & $1.3 \%$ & $0.4 \%$ & $1.3 \%$ & & & & 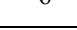 \\
\hline \multirow{2}{*}{62} & 212 & 224 & 225 & 228 & 353 & 12 & 25 & 50 \\
\hline & & $5.7 \%$ & $6.1 \%$ & $7.6 \%$ & נ.נכ & 1.2 & 2.5 & 5.9 \\
\hline \multirow{2}{*}{93} & 204 & 220 & 221 & 212 & 274 & 11 & 25 & 68 \\
\hline & & $7.8 \%$ & $8.3 \%$ & $3.9 \%$ & 21.4 & 1.1 & 2.0 & 0.0 \\
\hline \multirow{2}{*}{121} & 197 & 205 & 204 & 204 & 31.2 & 1.1 & 24 & 62 \\
\hline & & $4.1 \%$ & $3.6 \%$ & $3.6 \%$ & & & 2.7 & 0.2 \\
\hline
\end{tabular}

TABLE VII

PERFormance VALIDATION IN 9-NODE TOPOLOGY WITH 22-CORE MCF

\begin{tabular}{|c|c|c|c|c|c|c|c|c|}
\hline \multicolumn{9}{|c|}{ 9-node Topology, $|\mathcal{C}|=22,|\mathcal{D}|=800$} \\
\hline \multirow[b]{2}{*}{$\boldsymbol{\eta}$} & \multicolumn{4}{|c|}{$\Phi$} & \multicolumn{4}{|c|}{ Exec. Time (min) } \\
\hline & ILP1 & $\begin{array}{l}\text { SA- } \\
\text { LISA }\end{array}$ & $\begin{array}{l}\text { SA- } \\
\text { BLSA }\end{array}$ & $\begin{array}{l}\text { SA- } \\
\text { MRSA }\end{array}$ & ILP1 & $\begin{array}{l}\text { SA- } \\
\text { LISA }\end{array}$ & $\begin{array}{l}\text { SA- } \\
\text { BLSA }\end{array}$ & $\begin{array}{l}\text { SA- } \\
\text { MRSA }\end{array}$ \\
\hline 0 & $\begin{array}{l}215 \\
6 \%\end{array}$ & 204 & 204 & 203 & $>12 \mathrm{~h}$ & 1.2 & 2.3 & 5 \\
\hline 24 & $\begin{array}{l}205 \\
11 \% \\
\end{array}$ & 193 & 198 & 192 & $>12 \mathrm{~h}$ & 2 & 2.3 & 4.5 \\
\hline 48 & 179 & $\begin{array}{r}185 \\
3.4 \% \\
\end{array}$ & $\begin{array}{r}190 \\
6.2 \% \\
\end{array}$ & $\begin{array}{c}186 \\
3.9 \% \\
\end{array}$ & 561.9 & 2.1 & 2.2 & 4.4 \\
\hline 72 & -- & 178 & 188 & 181 & $>12 \mathrm{~h}$ & 2.5 & 2.2 & 3.7 \\
\hline 96 & 168 & $\begin{array}{r}176 \\
4.8 \% \\
\end{array}$ & $\begin{array}{r}179 \\
6.6 \% \\
\end{array}$ & $\begin{array}{c}177 \\
5.4 \% \\
\end{array}$ & 206.3 & 1.2 & 2.2 & 3.7 \\
\hline
\end{tabular}

consumption of MIMO-DSP, we can look for $\eta$ values lower than sweet spot, even requiring moderately higher spectral resources. It should be assessed what is more economically attractive: whether to implement MIMO to increase GoS or to save MIMO costs at expenses of sacrificing network GoS.

\section{MIMO equalization and Spectral Requirements Benefits}

This subsection aims to evaluate the benefits in terms of spectrum savings that MIMO equalization offers. To this end, we employ the proposed meta-heuristics to illustrate quantitative results in the larger DT12 and NSF15 networks. Fig. 2 (a-d) show the spectrum usage $(\Phi)$ versus the number of lightpaths with MIMO $(\eta)$. In line with the results previously shown in Tab. IV to VII, we start from the MCF-enabled solution without MIMO $(\eta=0)$ until finding the sweet spot $x$ (where the MF baseline is reached), plotting several intermediate points. This MF baseline was obtained considering candidate lightpaths only employing $m_{l^{(1)}}$ modulation formats (i.e., with MIMO) or, what is the same, considering the OSNR as the only TR limiting factor. Note that this situation would occur if the input parameter $\eta$ of heuristics is equal to $|\mathcal{D}|$. In turn, $\eta$ should be modified with an appropriate granularity to obtain an accurate sweet spot. For this purpose, for each meta-heuristic we plot several points $(\eta, \Phi)$ and the trend line over them.

Fig. $2(\mathrm{a}, \mathrm{b}, \mathrm{c})$ show the results in the DT12 network with $|\mathcal{C}|$ equal to 19,22 and 30 , respectively, for 1000 offered demands. For $|\mathcal{C}|=19$, the number of demands that are candidates to be served with MIMO ( $\left.|\mathcal{D}|_{\text {MiMo_Candidates }}\right)$ is 650. As observed, in spite of the ICXT, the sweet spot is lower than $|\mathcal{D}|$ and $|\mathcal{D}|_{\text {MiMo_Candidates, close to } 360 \text {, which }}$ 

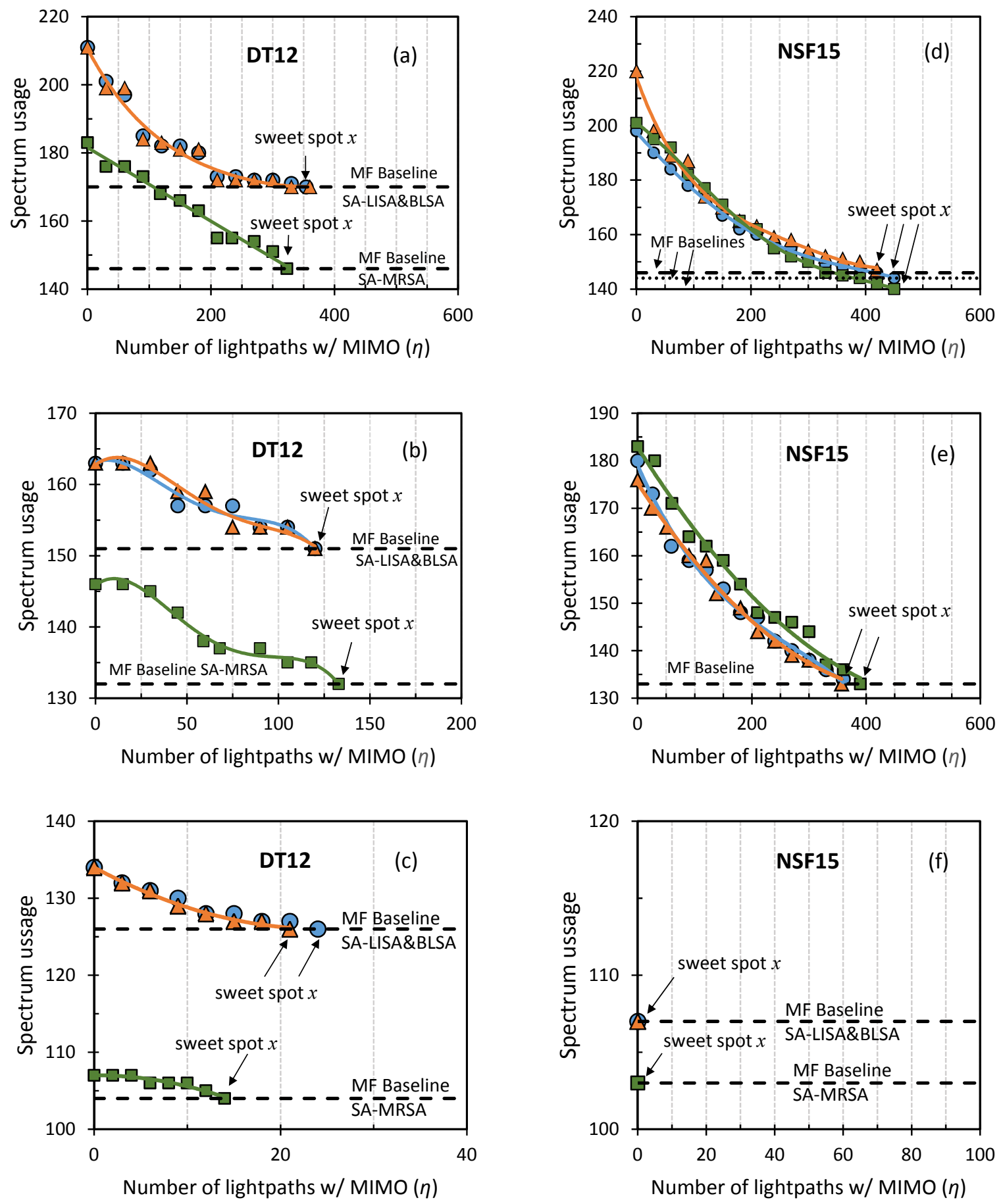

\begin{tabular}{|cc|}
\hline O SA-LISA & $\triangle$ SA-BLSA \\
$\square$ SA-MRSA & -- MF Baseline \\
\hline
\end{tabular}

Fig. 2. Spectrum usage versus number of MIMO lightpaths for: (a) DT12 network with 19-core MCF, (b) DT12 network with 22 -core MCF, (c) DT12 network with 30-core MCF (d) NSF15 network with 19-core MCF, (e) NSF15 network with 22-core MCF, and (f) NSF15 network with 30-core MCF.

represents the $36 \%(360 / 1000)$ of the total number of established lightpaths. For $|\mathcal{C}|=22$, the number of demands that are candidates to be served with MIMO is equal to 345 and the sweet spot $x$ is close to 120 , which represents the $12 \%$ of the total number of established lightpaths. Finally, for $|\mathcal{C}|=30,|\mathcal{D}|_{\text {MiMo_Candidates }}$ is equal to 72 and the sweet spot $x$ is around 24 representing the $2.4 \%$ of total established lightpaths. As observed, SA-LISA and SA-BLSA metaheuristics achieve very similar performance (in terms of spectrum usage) for different $\eta$ values, while SA-MRSA outperforms both of them by approximately 10-20\%. Observe that in Fig. 2 (a,b,c) are depicted two MF baselines (for SA- 
LISA\&BLSA and SA-MRSA, respectively). The reason behind this behavior is that the diameter and the good connectivity (see values from Tab. I obtained by natural connectivity definition presented in [42]) of the DT12 network allow SA-MRSA metaheuristic exploring longer paths for $|\mathcal{C}|=19,|\mathcal{C}|=22$ and $|\mathcal{C}|=30$ even in absence of MIMO equalization. Recall that MRSA heuristic explores disjointpaths in order to maximize the reuse of FS indexes. The higher the number of feasible disjoint paths, the higher the spectrum reutilization. Note that this behavior was not observed in the 6-node topology (used in the previous subsection, which has also similar network diameter but lower network connectivity), since in this short topology (in terms of network nodes and links) there are not so many possibilities to find disjoint paths. Consequently, the results reveal that the performance of the SA-MRSA meta-heuristic depends on the network diameter and connectivity.

Fig. $2(\mathrm{~d}, \mathrm{e}, \mathrm{f})$ show the results for NSF15 network with $|\mathcal{C}|$ equal to 19,22 and 30 , respectively, for 800 offered demands. For $|\mathcal{C}|=19$, the number of demands that are candidates to be served with MIMO ( $\left.|\mathcal{D}|_{\text {MiMo_Candidates }}\right)$ is 625 . As observed, in spite of the negative effects of the ICXT in a larger network size (NSF15 vs. DT12), the sweet spot is lower than $|\mathcal{D}|$ and $|\mathcal{D}|_{\text {MiMo Candidates. In fact, we have found that sweet spot } x \text { is }}$ close to 450 , which represents the $56 \%(450 / 800)$ of the total number of established lightpaths. For $|\mathcal{C}|=22$, the number of demands that are candidates to be served with MIMO is equal to 650 and the sweet spot $x$ is close to 360 representing the $45 \%$ of the total number established lightpaths. Finally, for $|\mathcal{C}|=30,|\mathcal{D}|_{\text {MiMo_Candidates }}$ is equal to only 3 , and there is no need for MIMO equalization ( $0 \%$ MIMO requirement). This result confirms previous findings presented in [24] about ICXT impact. The fact is that ICXT impact depends on a combination of several topological characteristics (network diameter, average path length, network connectivity, etc.) and traffic distribution. In our scenario, considering the NSF15 network without MIMO, almost all transmission distances use the same modulation formats as with MIMO equalization. This is the reason why only 3 demands are candidates to be served with MIMO, which do not affect the spectrum usage at all. Moreover, for each meta-heuristic we also plot several points $(\eta, \Phi)$ and the trend line over them. As observed, for $|\mathcal{C}|=19$ the three meta-heuristics SA-LISA, SA-BLSA and SA-MRSA, experiment similar performance with some differences between MF baseline of each meta-heuristic. Conversely to what happens in the DT12 network, SA-MRSA does not outperform the other options so significantly. The reason is that the diameter of NSF15 with $|\mathcal{C}|=19,|\mathcal{C}|=22$ or $|\mathcal{C}|=30$, hinders exploring feasible disjoint paths (recall that we consider transparent transmission) in order to maximize the reutilization of FSs.

Regarding the execution times, as in the previous subsection, the lowest execution times are reported for SALISA, while the highest ones are those of SA-MRSA. There is no case where longer time than $1.8 \mathrm{~min}$ and $7.4 \mathrm{~min}$ is reported for SA-LISA and SA-MRSA, respectively. Likewise, SA-BLSA experiments intermediate execution times between SA-LISA and SA-MRSA meta-heuristics. It is worth highlighting the low execution times of all lightweight meta- heuristics, demonstrating their suitability to solve the RMMSA problem even in large-scale instances.

Finally, in order to save on computational complexity and power consumption of MIMO-DSP we can look for $\eta$ values lower than sweet spot. For example, for the best results of Fig. 2 (a-e) a $50 \%$ of MIMO reduction is translated into a $5-15 \%$ of penalty in spectrum usage. That is, the spectral requirements evidences a slow decrement as the $\eta$ increases, as highlighted when analyzing ILP1 results in previous subsection.

\section{MIMO complexity and Crosstalk Compensation}

The effectiveness of MIMO-DSP to undo crosstalk mainly depends on the filter characteristics and some physical layer impairments. For instance, in case of Multi-Mode Fibers (MMFs), the number of taps per carrier increases with the Differential Mode Delay (DMD) and transmission distance. This, as stated in [43], leads to a hard convergence and an unstable adaptation of the MIMO equalizer. As a result, in the presence of relevant DMD, regardless of the filter length, crosstalk remains after MIMO equalization. Since stronglycoupled MCFs are considered a form of MMFs (equivalent to one core with several so-called super-modes) [22], for this SDM fiber, the impact of DMD on the complexity and effectiveness of MIMO-DSP is also relevant. Taking as an example the experiment carried out in [16], six column filters with 100 taps were needed to remove a $-4 \mathrm{~dB}$ accumulated crosstalk almost completely in a 3-core strongly-coupled $\mathrm{MCF}$, after $24 \mathrm{~km}$ of propagation for a target $10^{-3} \mathrm{BER}$. This conclusion suggests us that a remaining crosstalk after MIMO equalization, although small, was evidenced.

As in weakly-coupled MCFs all cores are not coupled together, while DMD can be negligible under certain conditions [44], the MIMO-DSP complexity can be relaxed by reducing the number of filters as well as their length, respectively. To illustrate how the number of filters can be reduced, let us consider the 7-core MCF depicted in Fig. 3(a). The center core \#1 is coupled with 6 neighboring cores, while the outer cores from $\# 2$ to $\# 7$ are only coupled with 3 cores. Therefore, a full $7 \times 7$ MIMO (without polarization decoupling) would not be needed to compensate ICXT, but rather the MIMO equalizer matrix shown in Fig. 3(b) would be enough, where $h$ accounts for the adaptation coefficient of the filter in position $i, j$. Core \#1 is equalized with all the rest of the cores, core \#2 is only equalized with cores \#1, \#3, and \#7; and so on. As a result, instead of 49 (7x7) filters, only 31 $(7+6 \cdot 4)$ would be enough, which means a $\sim 37 \%$ complexity reduction in terms of the number of filters. In general, we can compute the required number of filters of a MIMO equalizer as $\sum_{q=1}^{|\mathcal{C}|}$ (number of neighboring $\operatorname{cores}_{q}+1$ ). Applying the same analysis for the 19-core MCF prototype (cf. Fig. 1(a) in [31]), the number of required filters would be 91 against 361 (19x19), i.e., $\sim 75 \%$ of complexity reduction.

On the other hand, non-ideal MIMO compensation in weakly-coupled MCFs could be assumed for those desirable lightpaths to be equalized, either due to intrinsic limitations of MIMO-DSP or an intentional relaxation to further reduce its complexity. In such a case, the remaining crosstalk might be so low to let network performance totally unaffected or, even affecting it to a certain degree, it might suffice to approach the 
(a)

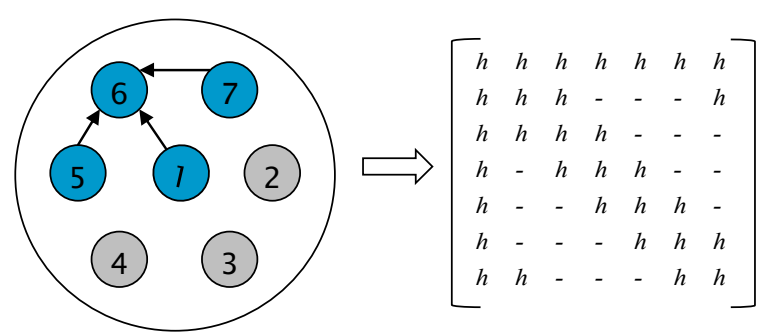

Fig. 3. 7-core MCF: (a) cross-sectional view (b) MIMO equalizer matrix

performance of an equivalent crosstalk-free SDM fiber solution. For instance, for the same 19-core MCF prototype [33], if we concentrate on the ICXT reduction between the center core $\# 1$ and its neighbors from $\# 2$ to $\# 7$, again the same MIMO equalizer matrix of Fig. 3(b) should be considered instead of a full $7 \times 7$ MIMO. In such a case, after applying MIMO to these cores from $\# 1$ to $\# 7$, the crosstalk compensation margin (per km) should be around $3 \mathrm{~dB}$, since the worst aggregate ICXT now will be reported for outer cores from \#8 to \#19 (having 3 neighboring cores each), where the aggregate crosstalk is around $-43 \mathrm{~dB}$ per $30 \mathrm{~km}$ (i.e., -57.8 $\mathrm{dB} / \mathrm{km}$ instead of $-54.8 \mathrm{~dB} / \mathrm{km}$ ). This reduces the complexity (in terms of the number of filters) by around $37 \%$ and $91 \%$ versus full $7 \times 7$ and $19 \times 19$ MIMO, respectively.

The previous results presented in subsections V.B and V.C were obtained considering ideal MIMO able to completely suppress the ICXT. In this subsection, we aim to evaluate nonideal MIMO by assuming a compensation margin of crosstalk from $1 \mathrm{~dB}$ to $6 \mathrm{~dB}$ per $1 \mathrm{~km}$ of fiber propagation, which correspond to a $20-75 \%$ of coupled power reduction. Note that, from the experiment in [16], much larger compensation margins should be obtained to almost completely suppress a large $-4 \mathrm{~dB}$ accumulated crosstalk. For this set of experiments, Algorithm 2 and 3 for different heuristics are the same, only changing the reference scenario in the selection of the MSE modulation format with MIMO $m_{l^{(1)}}$, therefore, the fitness function $f$ and the reference value $V$. This means that our proposal of minimizing the number of lightpaths with MIMO aiming to improve the network throughput is valid even in case of non-ideal MIMO equalization.

Specifically, in this subsection we consider the MCF prototype with the worst aggregate ICXT, namely, the 19-core MCF with the same set of demands $\mathcal{D}$ used in previous subsection V.C. Fig. $4(\mathrm{a}, \mathrm{b})$ show the spectrum usage $(\Phi)$ as a function of the number of lightpaths with MIMO equalization $(\eta)$ using SA-MRSA for DT12 and NSF15 networks, respectively. In each graph, three curves are shown for three compensation crosstalk margins, namely, $1 \mathrm{~dB} / \mathrm{km}, 3 \mathrm{~dB} / \mathrm{km}$ and $6 \mathrm{~dB} / \mathrm{km}$. As observed, in the DT12 network, results shown in Fig. 4(a) disclose that $1 \mathrm{~dB} / \mathrm{km}$ and $3 \mathrm{~dB} / \mathrm{km}$ crosstalk compensation do not reach the MF baseline, demanding $6 \mathrm{~dB} / \mathrm{km}$ crosstalk compensation to reach it. Indeed, $6 \mathrm{~dB} / \mathrm{km}$ crosstalk compensation yields the same results as the ones depicted in Fig. 2(a). This means that, the impact of ICXT below $-60 \mathrm{~dB} / \mathrm{km}$ (not included) is irrelevant (as also corroborated in [45]). Initially, the performance gap between the considered MCF- and equivalent MF-based solution is $\sim 25 \%$ (183/146) without MIMO. Then, by applying
MIMO-DSP yielding $1 \mathrm{~dB} / \mathrm{km}$ crosstalk compensation to $\sim 13 \%(125 / 1000)$ of the lightpaths, the performance gap is reduced to $\sim 18 \%$. Meanwhile, for a MIMO-DSP yielding 3 $\mathrm{dB} / \mathrm{km}$ crosstalk compensation, this gap downs to $\sim 4 \%$ when $\sim 28 \%(275 / 1000)$ lightpaths are equalized. Note that, similarly to the sweet spot $x$, curves for $1 \mathrm{~dB} / \mathrm{km}$ and $3 \mathrm{~dB} / \mathrm{km}$ of crosstalk compensation present a point $\eta_{i}$, beyond which the spectrum usage does not decrease, since more equalized lightpaths do not provide any spectrum savings.
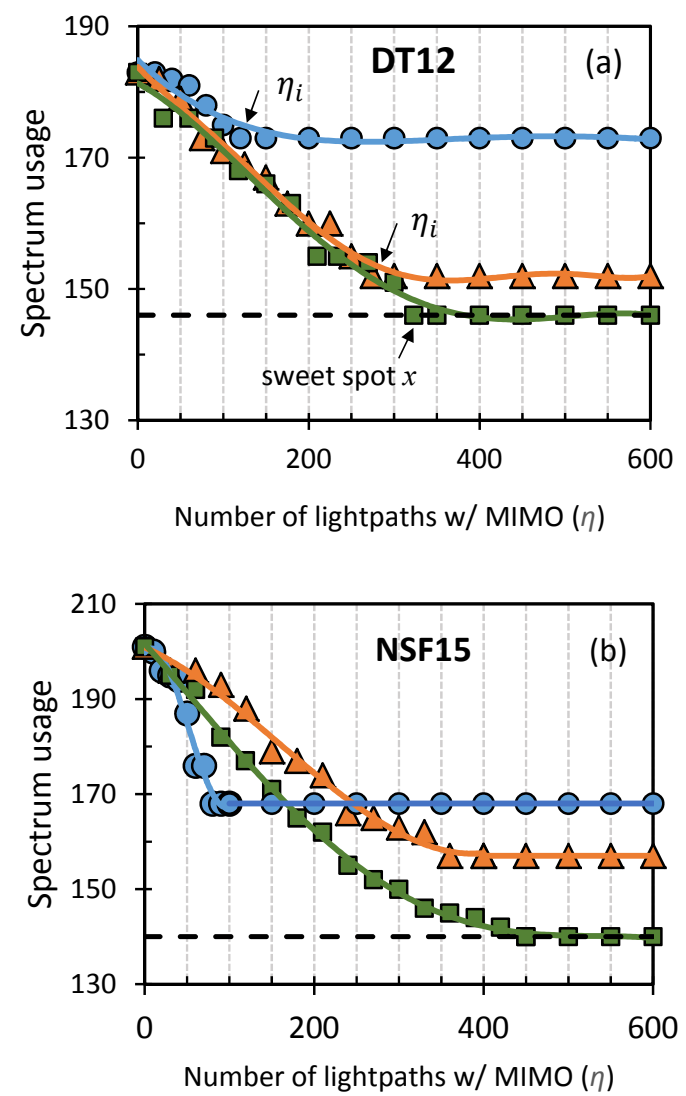

\begin{tabular}{|cl|}
\hline 0 & 1-dB ICXT_comp. \\
$\Delta$ & 3-dB ICXT_comp. \\
$\square$ & 6-dB ICXT_comp. \\
\hline
\end{tabular}

Fig. 4. Spectrum usage vs. number of lightpaths with $1-\mathrm{dB} / \mathrm{km}, 3-\mathrm{dB} / \mathrm{km}$ and 6-dB/km MIMO ICXT compensation in the (a) DT12 (b) NSF15 network.

As for the NSF15 network, results shown in Fig. 4(b) are slightly different, especially with $1 \mathrm{~dB} / \mathrm{km}$ crosstalk compensation. This crosstalk compensation margin seems yielding better spectrum usage benefits than $6 \mathrm{~dB} / \mathrm{km}$ before reaching $\eta_{i}$. The fact is that, the higher the crosstalk compensation, the larger $|\mathcal{D}|_{\text {MiMo_Candidates }}$, which hinders the search of global optimum solutions in a polynomial time. For example, for $1 \mathrm{~dB} / \mathrm{km}$ crosstalk compensation,

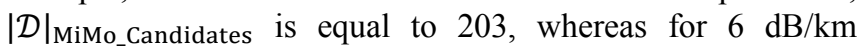
$|\mathcal{D}|_{\text {Mimo_Candidates }}$ raises up to 625 . In particular, the initial performance gap between the considered MCF- and equivalent MF-based solution decreases from $\sim 44 \%$ to $\sim 20 \%$ and $\sim 12 \%$ when MIMO-DSP yielding $1 \mathrm{~dB} / \mathrm{km}$ and $3 \mathrm{~dB} / \mathrm{km}$ crosstalk compensation is applied to $21 \%(168 / 800)$ and $45 \%(360 / 800)$ 
of lightpaths, respectively. Finally, again a $6 \mathrm{~dB} / \mathrm{km}$ crosstalk compensation would be enough to reach the MF baseline.

According to the results of subsection V.C and V.D, the performance of a crosstalk-free SDM solution can be met even in case of non-ideal MIMO. For this purpose, the remaining crosstalk has to be lower than $-60 \mathrm{~dB} / \mathrm{km}$ (not included). That is, around 6 and $4 \mathrm{~dB} / \mathrm{km}$ of crosstalk compensation is enough for the 19- and 22-core MCFs, respectively, considered in this work.

Finally, even with the potential complexity reduction of MIMO-DSP in weakly-coupled MCFs against stronglycoupled ones, real-time DSP implementation is still an open question for long-haul communications. Nevertheless, techniques like DMD fiber management [46], [47], unconstrained frequency-domain equalization to accelerate the convergence during filter adaptation against time-domain one [18], or bit-width reduction in floating point operations of Field Programmable Gate Arrays (FPGAs)/ApplicationSpecific Integrated Circuits (ASICs) [48] foresee successful real-time DSP equalization. In fact, the first real-time $6 \times 6$ MIMO-DSP SDM transmission experiment was carried out in 2015 [49] for 3-core strongly-coupled MCF. The challenge for researchers is to continue putting efforts in key aspects that lead to further complexity reductions aimed at supporting realtime MIMO-DSP in backbone networks.

\section{CONCLUSIONS AND Future WORK}

In this work, we have studied an optimal MIMO assignment to mitigate the negative ICXT effects in MCF-enabled networks. If JoS is applied for channel routing, then the space resource allocation disappears and the lightpath allocation is simplified to the traditional RMSA problem for elastic optical networks. We have proposed to introduce the problem of MIMO assignment for a specific lightpath as an additional subproblem of the RMSA, calling this new problem as Route, Modulation format, MIMO and Spectrum Assignment (RMMSA). To optimally solve small RMMSA problem instances, we have proposed an ILP formulation. Besides, a SA-based meta-heuristic making use of three different greedy heuristics (LISA, BLSA, MRSA) has also been presented to solve large problem instances. The accumulated ICXT can negatively affect the performance of long-haul communications enabled by weakly-coupled MCFs. To address this issue our work has been concentrated on applying MIMO equalization to the least amount of lightpaths, where no so high order MIMO is expected to counteract or cancel the ICXT effect. Assuming ideal MIMO compensation, our results reveal that in national backbone networks up to $36 \%$ lightpaths require MIMO-based ICXT suppression in order to match the spectral requirements of a crosstalk-free SDM solution (e.g. parallel single mode fibers). Meanwhile, for continental backbone networks, up to $56 \%$ of lightpaths need MIMO equalization. In the case of non-ideal MIMO, considering e.g., a $3 \mathrm{~dB} / \mathrm{km}$ crosstalk compensation, these percentages decrease to $28 \%$ and $45 \%$, respectively, with up to $12 \%$ of performance gap versus the MF benchmark scenario. In any case, a trade-off between MIMO-DSP complexity and GoS has to be analyzed in detail to propose an overall MIMO solution. For instance, we could assess what is more economically attractive: whether to implement MIMO to increase GoS or to save MIMO costs at expenses of sacrificing $\mathrm{GoS}$, which we pretend to address in future works. Additional works can be focused on analyzing the MIMO assignment problem for other super-channel configurations and switching schemes.

\section{ACKNOWLEDGMENTS}

This work has been partially supported by the Spanish Ministry of Economy and Competitiveness under contracts TEC2017-90034-C2-1-R and TEC2017-90034-C2-2-R that receive funding from FEDER.

Rubén Rumipamba is recipient of a full scholarship from Secretaría Nacional de Ciencia y Tecnología (SENESCYT) Ecuador (2015-AR2Q9065).

\section{REFERENCES}

[1] S. K. Korotky, "Price-Points for Components of Multi-Core Fiber Communication Systems in Backbone Optical Networks," J. Opt. Commun. Netw., vol. 4, no. 5, p. 426, 2012.

[2] Y. Li, N. Hua, and X. Zheng, "CapEx advantages of multicore fiber networks," Photonic Netw. Commun., vol. 31, no. 2, pp. 228-238, 2016.

[3] T. Hayashi, T. Taru, O. Shimakawa, T. Sasaki, and E. Sasaoka, "Ultra-low-crosstalk multi-core fiber feasible to ultra-long-haul transmission," 2011 Opt. Fiber Commun. Conf. Expo. Natl. Fiber Opt. Eng. Conf., pp. 1-3, 2011.

[4] T. Mizuno, K. Shibahara, F. Ye, Y. Sasaki, Y. Amma, K. Takenaga, Y. Jung, K. Pulverer, H. Ono, Y. Abe, M. Yamada, K. Saitoh, S. Matsuo, K. Aikawa, M. Bohn, D. J. Richardson, Y. Miyamoto, and T. Morioka, "Long-Haul Dense Space-Division Multiplexed Transmission over LowCrosstalk Heterogeneous 32-Core Transmission Line Using a Partial Recirculating Loop System," J. Light. Technol., vol. 35, no. 3, pp. 488-498, 2017.

[5] O. Gerstel, M. Jinno, A. Lord, and S. J. Ben Yoo, "Elastic optical networking: A new dawn for the optical layer?," IEEE Commun. Mag., vol. 50, no. 2, pp. 12-20, 2012.

[6] A. Muhammad, G. Zervas, and R. Forchheimer, "Resource Allocation for Space-Division Multiplexing: Optical White Box Versus Optical Black Box Networking," J. Light. Technol., vol. 33, no. 23, pp. 4928-4941, Dec. 2015.

[7] Y. Zhao, Y. Zhu, C. Wang, X. Yu, C. Liu, B. Liu, and J. Zhang, "Super-channel oriented routing, spectrum and core assignment under crosstalk limit in spatial division multiplexing elastic optical networks," Opt. Fiber Technol., vol. 36, pp. 249-254, 2017.

[8] H. Tode and Y. Hirota, "Routing, Spectrum, and Core and/or Mode Assignment on Space-Division Multiplexing Optical Networks [Invited]," J. Opt. Commun. Netw., vol. 9, no. 1, p. A99, Jan. 2017.

[9] R. Rumipamba-Zambrano, J. Perelló, J. M. Gené, and S. Spadaro, "Cost-effective spatial super-channel allocation in Flex-Grid/MCF optical core networks," Opt. Switch. Netw., vol. 27, pp. 93-101, Jan. 2018.

[10] D. Klonidis, F. Cugini, O. Gerstel, M. Jinno, V. Lopez, E. Palkopoulou, M. Sekiya, D. Siracusa, G. Thouénon, and C. Betoule, "Spectrally and spatially flexible optical network planning and operations," IEEE Commun. Mag., vol. 53, no. 2, pp. 69-78, 2015.

[11] M. D. Feuer, L. E. Nelson, X. Zhou, S. L. Woodward, R. Isaac, Benyuan Zhu, T. F. Taunay, M. Fishteyn, J. M. Fini, and M. F. Yan, "Joint Digital Signal Processing Receivers 
for Spatial Superchannels," IEEE Photonics Technol. Lett., vol. 24, no. 21, pp. 1957-1960, Nov. 2012.

[12] B. Shariati, A. Mastropaolo, D. Klonidis, and I. Tomkos, "Cost Benefit Quantification of SDM Network Implementations based on Spatially Integrated Network Elements," 42nd Eur. Conf. Exhib. Opt. Commun., no. M.1.F.4, pp. 82-84, 2016.

[13] L. E. Nelson, M. D. Feuer, K. Abedin, X. Zhou, T. F. Taunay, J. M. Fini, B. Zhu, R. Isaac, R. Harel, G. Cohen, and D. M. Marom, "Spatial superchannel routing in a twospan ROADM system for space division multiplexing," $J$. Light. Technol., vol. 32, no. 4, pp. 783-789, 2014.

[14] A. M. Ortiz and R. L. Sáez, "Multi-Core Optical Fibers: Theory, Applications and Opportunities," Sel. Top. Opt. Fiber Technol. Appl., no. February, 2018.

[15] T. Tanaka, K. Pulverer, U. Habel, C. Castro, M. Bohn, T. Mizuno, A. Isoda, K. Shibahara, T. Inui, Y. Miyamoto, Y. Sasaki, Y. Amma, K. Aikawa, S. Jain, Y. Jung, S. Alam, D. J. Richardson, M. Nooruzzaman, and T. Morioka, "Demonstration of Single-Mode Multicore Fiber Transport Network with Crosstalk-Aware In-Service Optical Path Control," J. Light. Technol., vol. 8724, no. c, 2017.

[16] R. Ryf, R. J. Essiambre, S. Randel, A. H. Gnauck, P. J. Winzer, T. Hayashi, T. Taru, and T. Sasaki, "MIMO-based crosstalk suppression in spatially multiplexed $3 \times 56-\mathrm{Gb} / \mathrm{s}$ PDM-QPSK signals for strongly coupled three-core fiber," IEEE Photonics Technol. Lett., vol. 23, no. 20, pp. 1469 1471, 2011.

[17] Y. Li, N. Hua, and X. Zheng, "Routing, wavelength and core allocation planning for multi-core fiber networks with MIMO-based crosstalk suppression," 2015 Opto-Electronics Commun. Conf. OECC 2015, vol. 2, pp. 2-4, 2015.

[18] S. Randel, P. J. Winzer, M. Montoliu, and R. Ryf, "Complexity Analysis of Adaptive Frequency-Domain Equalization for MIMO-SDM Transmission," 39th Eur. Conf. Exhib. Opt. Commun. (ECOC 2013), pp. 801-803, 2013.

[19] Y. Li, N. Hua, and X. Zheng, "A Capacity Analysis for Space Division Multiplexing Optical Networks with MIMO Equalization," Opt. Fiber Commun. Conf., p. Th2A.15, 2017.

[20] N. P. Diamantopoulos, B. Shariati, and I. Tomkos, "On the Power Consumption of MIMO Processing and its Impact on the Performance of SDM Networks," Opt. Fiber Commun. Conf., p. Th2A.18, 2017.

[21] P. J. Winzer, "Spatial Multiplexing: The Next Frontier in Network Capacity Scaling," Eur. Conf. Exhib. Opt. Commun., p. We.1.D.1, 2013.

[22] K. Saitoh and S. Matsuo, "Multicore fiber technology," $J$ Light. Technol., vol. 34, no. 1, 2016.

[23] T. Hayashi, T. Taru, O. Shimakawa, T. Sasaki, and E. Sasaoka, "Design and fabrication of ultra-low crosstalk and low-loss multi-core fiber," Opt. Express, vol. 19, no. 17, p. 16576, 2011.

[24] R. Rumipamba-Zambrano, J. Perelló, J. M. Gené, and S. Spadaro, "Capacity quantification of joint-switching-enabled flex-grid/SDM optical backbone networks," in 2017 Optical Fiber Communications Conference and Exhibition, OFC 2017 - Proceedings, 2017.

[25] B. J. Puttnam, R. S. Luis, W. Klaus, J. Sakaguchi, J.-M. Delgado Mendinueta, Y. Awaji, N. Wada, Y. Tamura, T. Hayashi, M. Hirano, and J. Marciante, "2.15 Pb/s transmission using a 22 core homogeneous single-mode multi-core fiber and wideband optical comb," in 2015 European Conference on Optical Communication (ECOC), 2015, pp. 1-3.
[26] International Telecommunication Union - ITU-T, "G.694.1 (02/2012), Spectral grids for WDM applications: DWDM frequency grid,"Ser. G.694.1, pp. 1-16, 2012.

[27] K. Christodoulopoulos, I. Tomkos, and E. a Varvarigos, "Elastic Bandwidth Allocation in Flexible OFDM- based Optical Networks," J. Light. Technol., vol. 29, no. 9, pp. 1354-1366, 2011.

[28] M. Klinkowski and K. Walkowiak, "Routing and spectrum assignment in spectrum sliced elastic optical path network," IEEE Commun. Lett., vol. 15, no. 8, pp. 884-886, 2011.

[29] Y. Wang, X. Cao, and Y. Pan, "A study of the routing and spectrum allocation in spectrum-sliced Elastic Optical Path networks," Proc. - IEEE INFOCOM, pp. 1503-1511, 2011.

[30] S. Kirkpatrick, C. D. Gelatt, and M. P. Vecchi, "Optimization by simulated annealing.," Science, vol. 220, no. 4598 , pp. 671-80, May 1983.

[31] J. Sakaguchi, Y. Awaji, N. Wada, A. Kanno, T. Kawanishi, T. Hayashi, T. Taru, T. Kobayashi, and M. Watanabe, "Space division multiplexed transmission of $109-\mathrm{Tb} / \mathrm{s}$ data signals using homogeneous seven-core fiber," J. Light. Technol., vol. 30, no. 4, pp. 658-665, 2012.

[32] A. Sano, H. Takara, T. Kobayashi, H. Kawakami, H. Kishikawa, T. Nakagawa, Y. Miyamoto, Y. Abe, H. Ono, K. Shikama, M. Nagatani, T. Mori, Y. Sasaki, I. Ishida, K. Takenaga, S. Matsuo, K. Saitoh, M. Koshiba, M. Yamada, H. Masuda, and T. Morioka, "409-Tb/s $+409-\mathrm{Tb} / \mathrm{s}$ crosstalk suppressed bidirectional MCF transmission over $450 \mathrm{~km}$ using propagation-direction interleaving.," Opt. Express, vol. 21, no. 14, pp. 16777-16783, 2013.

[33] J. Sakaguchi, W. Klaus, B. J. Puttnam, J. M. D. Mendinueta, Y. Awaji, N. Wada, Y. Tsuchida, K. Maeda, M. Tadakuma, K. Imamura, R. Sugizaki, T. Kobayashi, Y. Tottori, M. Watanabe, and R. V Jensen, "19-core MCF transmission system using EDFA with shared core pumping coupled via free-space optics.," Opt. Express, vol. 22, no. 1, pp. 90-5, 2014.

[34] Y. Amma, Y. Sasaki, K. Takenaga, S. Matsuo, J. Tu, K. Saitoh, M. Koshiba, T. Morioka, and Y. Miyamoto, "Highdensity Multicore Fiber with Heterogeneous Core Arrangement," in Optical Fiber Communication Conference, 2015, p. Th4C.4.

[35] R. Rumipamba-Zambrano, F.-J. Moreno-Muro, J. Perelló, P. Pavón-Mariño, and S. Spadaro, "Space continuity constraint in dynamic Flex-Grid/SDM optical core networks: An evaluation with spatial and spectral super-channels," Comput. Commun., vol. 126, 2018.

[36] K. Walkowiak, M. Klinkowski, and P. Lechowicz, "Dynamic Routing in Spectrally Spatially Flexible Optical Networks with Back-to-Back Regeneration," J. Opt. Commun. Netw., vol. 10, no. 5, p. 523, May 2018.

[37] P. Poggiolini, G. Bosco, A. Carena, V. Curri, Y. Jiang, and F. Forghieri, "The GN-Model of Fiber Non-Linear Propagation and its Applications," J. Light. Technol., vol. 32, no. 4, pp. 694-721, Feb. 2014

[38] P. J. Winzer, a. H. Gnauck, a. Konczykowska, F. Jorge, and J.-Y. Dupuy, "Penalties from in-band crosstalk for advanced optical modulation formats," 2011 37th Eur. Conf. Exhib. Opt. Commun., no. 1, pp. 1-3, 2011.

[39] B. Li, L. Gan, S. Fu, Z. Xu, M. Tang, W. Tong, and P. P. Shum, "The Role of Effective Area in the Design of Weakly Coupled MCF: Optimization Guidance and OSNR Improvement," IEEE J. Sel. Top. Quantum Electron., vol. 22, no. 2, 2016.

[40] M. N. Dharmaweera, L. Yan, M. Karlsson, and E. Agrell, "Nonlinear-Impairments- and Crosstalk-Aware Resource Allocation Schemes for Multicore-Fiber-based Flexgrid 
Networks," no. 1, pp. 1223-1225, 2016.

[41] IBM, "IBM ILOG CPLEX Optimizer." [Online]. Available: https://www.ibm.com/products/ilog-cplex-optimizationstudio.

[42] J. Wu, M. Barahona, Y. Tan, and H. Deng, "Robustness of random graphs based on graph spectra," Chaos An Interdiscip. J. Nonlinear Sci., vol. 22, no. 4, p. 043101, Dec. 2012.

[43] T. Mizuno, T. Kobayashi, H. Takara, A. Sano, H. Kawakami, T. Nakagawa, Y. Miyamoto, Y. Abe, T. Goh, M. Oguma, T. Sakamoto, Y. Sasaki, I. Ishida, K. Takenaga, S. Matsuo, K. Saitoh, and T. Morioka, "12-core x 3-mode Dense Space Division Multiplexed Transmission over $40 \mathrm{~km}$ Employing Multi-carrier Signals with Parallel MIMO Equalization," in Optical Fiber Communication Conference: Postdeadline Papers, 2014, p. Th5B.2.

[44] T. Sakamoto, T. Mori, M. Wada, T. Yamamoto, and T. Matsui, "Experimental and numerical evaluation of intercore differential mode delay characteristic of weaklycoupled multi-core fiber," vol. 22, no. 26, pp. 31966-31976, 2014.

[45] M. Klinkowski, P. Lechowicz, and K. Walkowiak, "A Study on the impact of inter-core crosstalk on SDM network performance," in IEEE Int. Conf. on Computing, Networking and Communications (ICNC), Maui, Hawaii, 2018.

[46] T. Sakamoto, T. Matsui, K. Saitoh, S. Saitoh, K. Takenaga, T. Mizuno, Y. Abe, K. Shibahara, Y. Tobita, S. Matsuo, K. Aikawa, S. Aozasa, K. Nakajima, and Y. Miyamoto, "Lowloss and Low-DMD Few-mode Multi-core Fiber with Highest Core Multiplicity Factor," vol. 1, pp. 20-22, 2016.

[47] T. Hayashi, Y. Tamura, T. Hasegawa, and T. Taru, "RecordLow Spatial Mode Dispersion and Ultra-Low Loss Coupled Multi-Core Fiber for Ultra-Long-Haul Transmission," $J$. Light. Technol., vol. 35, no. 3, pp. 450-457, 2017.

[48] R. G. H. Van Uden, C. M. Okonkwo, R. H. G. Van Uden, H. De Waardt, and A. M. J. Koonen, "The impact of bit-width reduced MIMO equalization for few mode fiber transmission systems," Eur. Conf. Opt. Commun. ECOC, pp. 2-4, 2014.

[49] S. Randel, S. Corteselli, D. Badini, D. Pilori, S. Caelles, S. Chandrasekhar, J. Gripp, H. Chen, N. K. Fontaine, R. Ryf, and P. J. Winzer, "First real-time coherent MIMO-DSP for six coupled mode transmission," 2015 IEEE Photonics Conf. IPC 2015, 2015. 